TRANSACTIONS OF THE

AMERICAN MATHEMATICAL SOCIETY

Volume 361, Number 7, July 2009, Pages 3633-3660

S 0002-9947(09)04644-3

Article electronically published on February 4, 2009

\title{
RECONSTRUCTION OF PATH ALGEBRAS FROM THEIR POSETS OF TILTING MODULES
}

\author{
DIETER HAPPEL AND LUISE UNGER
}

\begin{abstract}
Let $\Lambda=k \vec{\Delta}$ be the path algebra of a finite quiver without oriented cycles. The set of isomorphism classes of multiplicity free tilting modules is in a natural way a partially ordered set. We will show here that $\mathcal{T}_{\Lambda}$ uniquely determines $\vec{\Delta}$ if $\vec{\Delta}$ has no multiple arrows and no isolated vertices.
\end{abstract}

Let $\Lambda$ be a basic, connected finite dimensional algebra over an algebraically closed field $k$ and let $\bmod \Lambda$ be the category of finitely generated left $\Lambda$-modules. For a module $M \in \bmod \Lambda$ we denote by $\operatorname{pd}_{\Lambda} M$ the projective dimension of $M$ and by gl.dim $\Lambda$ the global dimension of $\Lambda$. A module $T \in \bmod \Lambda$ is called a tilting module if the following three conditions are satisfied: (i) $\operatorname{pd}_{\Lambda} T<\infty$, (ii) $\operatorname{Ext}_{\Lambda}^{i}(T, T)=0$ for all $i>0$ and (iii) there exists an exact sequence $0 \rightarrow{ }_{\Lambda} \Lambda \rightarrow T^{0} \rightarrow \cdots \rightarrow T^{r} \rightarrow 0$ with $T^{i} \in \operatorname{add} T$ for all $0 \leq i \leq r$, where add $T$ is the full subcategory of $\bmod \Lambda$ whose objects are direct sums of direct summands of $T$. We will say that a tilting module is basic or multiplicity free if in a direct sum decomposition of $T$ the indecomposable direct summands of $T$ occur with multiplicity one. Unless stated otherwise all tilting modules considered here will be assumed to be basic.

Tilting theory usually has two aspects. Originally it was used, which one might call the external aspect, to compare $\bmod \Lambda$ to $\bmod \operatorname{End}_{\Lambda} T$ for a $\Lambda$-tilting module $T$. The internal aspect, and this is the one we are interested in here, is to study tilting modules $T$ for a fixed $\Lambda$, and try to gather information about $\bmod \Lambda$.

Following $[\mathrm{AR}]$ we consider for a tilting module $T \in \bmod \Lambda$ the right perpendicular category

$$
T^{\perp}=\left\{X \in \bmod \Lambda \mid \operatorname{Ext}_{\Lambda}^{i}(T, X)=0 \text { for all } i>0\right\} .
$$

We consider the set $\mathcal{T}_{\Lambda}$ of all tilting modules over $\Lambda$ up to isomorphism. Note that $\mathcal{T}_{\Lambda}$ is always countable (compare for example $[\mathrm{H} 2$ ). In HU1 the following partial order $\leq$ on $\mathcal{T}_{\Lambda}$ was investigated. For $T, T^{\prime} \in \mathcal{T}_{\Lambda}$ we set $T \leq T^{\prime}$ provided $T^{\perp} \subset T^{\perp}$. One of the main results in [HU1] was that the Hasse quiver $\overrightarrow{\mathcal{K}}_{\Lambda}$ (i.e. the quiver of minimal inclusions) of this poset allows the following description: The vertices of $\overrightarrow{\mathcal{K}}_{\Lambda}$ are the elements of $\mathcal{T}_{\Lambda}$ and there is an arrow $T^{\prime} \rightarrow T$ in $\overrightarrow{\mathcal{K}}_{\Lambda}$ if $T^{\prime}=M \oplus X$ and

Received by the editors April 16, 2007.

2000 Mathematics Subject Classification. Primary 16G10, 16G70, 16E10.

The main results presented here were obtained while the authors were visiting the University of Sao Paulo and Shanghai Jiao Tong University. Both authors would like to thank their hosts Flavio Coelho and Pu Zhang for their hospitality. 
$T=M \oplus Y$ with $X, Y$ indecomposable, and there exists a non-split short exact sequence $0 \rightarrow X \rightarrow \tilde{M} \rightarrow Y \rightarrow 0$ with $\tilde{M} \in$ add $M$.

The main aim of this article is to investigate the following natural question. In which way does $\overrightarrow{\mathcal{K}}_{\Lambda}$ or $\mathcal{T}_{\Lambda}$ determine the isomorphism class of $\Lambda$ ? In general the answer to this question is negative. For example if $\Lambda$ is selfinjective, then $\overrightarrow{\mathcal{K}}_{\Lambda}$ consists of just a single vertex. In case $\Lambda$ is a hereditary algebra the question is more interesting. Since neither $\mathcal{T}_{\Lambda}$ nor $\overrightarrow{\mathcal{K}}_{\Lambda}$ will tell us whether or not $\Lambda$ has simple modules which are both projective and injective in case there are $n \geq 2$ simple modules (see section 3), we will assume that $\Lambda \simeq k \vec{\Delta}$ for a finite quiver without oriented cycles and without isolated vertices. This is trivially satisfied if $\vec{\Delta}$ is connected and has $n \geq 2$ vertices. The case that $\vec{\Delta}=0$ is of course trivial.

We will show in section 6 as a main result of this article that $\mathcal{T}_{\Lambda}$ uniquely determines $\vec{\Delta}$ if $\vec{\Delta}$ has no multiple arrows and no isolated vertices. Thus $\overrightarrow{\mathcal{K}}_{\Lambda}$ uniquely determines $\vec{\Delta}$ in case $\vec{\Delta}$ is a Dynkin quiver without isolated vertices. Note that in general it will be impossible to recover the precise number of arrows between two vertices in case of more than two arrows. Indeed, if $\vec{\Delta}_{r}$ is the two point quiver with $r \geq 2$ arrows and $\Lambda_{r}$ the path algebra of $\vec{\Delta}_{r}$ over $k$, then the partially ordered sets $\mathcal{T}_{\Lambda_{r}} \simeq \mathcal{T}_{\Lambda_{s}}$ for all $r, s \geq 2$. But we will show in section 6 that $\mathcal{T}_{\Lambda}$ contains the information as to whether or not there are multiple arrows in $\vec{\Delta}$ between a pair of vertices.

The article is organized as follows. In section 1 we recall from [H2 and [U1] some preliminary material about tilting modules. Section 2 deals with orthogonal categories in the sense of [GL]. There we will show that for a not necessarily hereditary algebra $\Lambda$ the poset $\mathcal{T}_{\Lambda}$ has an inductive property when forming orthogonal categories with respect to simple $\Lambda$-modules $S$ with $\operatorname{pd}_{\Lambda} S \leq 1$ and $\operatorname{id}_{\Lambda} S \leq 1$, where $\operatorname{id}_{\Lambda} M$ denotes the injective dimension of $M \in \bmod \Lambda$. Although this result will not be needed in the proof of the main theorem, we still think it is worthwhile to present here. In section 3 we will show how to determine the number of simple $\Lambda$-modules from $\overrightarrow{\mathcal{K}}_{\Lambda}$ for a connected hereditary algebra $\Lambda$. In section 4 we will study more closely the situation of Dynkin quivers and show that $\overrightarrow{\mathcal{K}}_{\Lambda}$ contains the information on the number of isomorphism classes of indecomposable modules. This easily allows us to deduce from $\overrightarrow{\mathcal{K}}_{\Lambda}$ the underlying Dynkin diagram. Section 5 contains some relevant information about the neighbors of ${ }_{\Lambda} \Lambda$ and of $D \Lambda_{\Lambda}$ in $\overrightarrow{\mathcal{K}}_{\Lambda}$. Section 6 then deals with the actual reconstruction of the orientation and the detection of multiple arrows of $\vec{\Delta}$.

We denote the composition of morphisms $f: X \rightarrow Y$ and $g: Y \rightarrow Z$ in a given category $\mathcal{K}$ by $f g$.

The notation and terminology introduced here will be fixed throughout this article. For unexplained representation-theoretic terminology we refer to $[\mathrm{R}]$ or ARS.

\section{Preliminaries}

We will briefly recall in this section some of the technical details and notation frequently used in the subsequent sections.

Let $\Lambda$ be a finite dimensional $k$-algebra over an algebraically closed field $k$. We denote by $n$ the number of simple $\Lambda$-modules and label them $S_{1}, \ldots, S_{n}$. Their 
projective covers (resp. injective envelopes) will be denoted by $P(1), \ldots, P(n)$ (resp. $I(1), \ldots, I(n))$. By $D$ we denote the standard duality with respect to $k$, so $D \Lambda_{\Lambda}=\bigoplus_{i=1}^{n} I(i)$.

We say that a module $M$ is a partial tilting module if $M$ is a direct summand of a tilting module. A partial tilting module $M$ with $n-1$ non-isomorphic indecomposable direct summands is called an almost complete partial tilting module. We point out that for a hereditary algebra $\Lambda$ any $\Lambda-$ module $N$ with $\operatorname{Ext}_{\Lambda}^{1}(N, N)=0$ is a partial tilting module (compare 4.1 in $[\mathrm{R}]$ ). This fact will be frequently used in the subsequent sections. Given a partial tilting module $M$ we say that $C$ is a complement to $M$ if $M \oplus C$ is a tilting module and add $M \cap$ add $C=0$. Note that a complement to an almost complete partial tilting module is indecomposable. If $T$ is a multiplicity free tilting module, we have the direct sum decomposition $T=\bigoplus_{i=1}^{n} T_{i}$. For $1 \leq i \leq n$ we denote it by $T[i]=\bigoplus_{j \neq i} T_{j}$. So $T[i]$ is an almost complete partial tilting module for $1 \leq i \leq n$. The following lemma (compare H2] ) is important

Lemma 1.1. Let $1 \leq i \leq n$. If $T[i]$ is not faithful, then $T_{i}$ is the unique (up to isomorphism) complement to $T[i]$. If $T[i]$ is faithful, then $T_{i}$ is either generated or cogenerated by $T[i]$.

(1) If $T_{i}$ is generated by $T[i]$ there exists a unique (up to isomorphism) indecomposable complement $X$ not isomorphic to $T_{i}$ and an exact sequence $0 \rightarrow X \rightarrow \widetilde{T[i]} \rightarrow T_{i} \rightarrow 0$ with $\widetilde{T[i]} \in \operatorname{add} T[i]$ such that $\operatorname{Ext}_{\Lambda}^{i}\left(X, T_{i}\right)=0$ for $i>0$ and $\operatorname{Ext}_{\Lambda}^{i}\left(T_{i}, X\right)=0$ for $i>1$.

(2) If $T_{i}$ is cogenerated by $T[i]$ there exists a unique (up to isomorphism) indecomposable complement $Y$ not isomorphic to $T_{i}$ and an exact sequence $0 \rightarrow T_{i} \rightarrow \widetilde{T[i]} \rightarrow Y \rightarrow 0$ with $\widetilde{T[i]} \in \operatorname{addT}[i]$ such that $\operatorname{Ext}_{\Lambda}^{i}\left(Y, T_{i}\right)=0$ for $i>1$ and $\operatorname{Ext}_{\Lambda}^{i}\left(T_{i}, Y\right)=0$ for $i>0$.

(3) If $\Lambda$ is hereditary, then the non-vanishing Ext-spaces in (2) and (3) are one dimensional.

(4) If $\Lambda$ is hereditary, then $T_{i}$ is never both generated and cogenerated by $T[i]$.

The exact sequences occurring in (1) and (2) will be called the exact sequences connecting the complements. We will also say that in the situation of (1) $T_{i}$ is replaced by the module $X$ or simply $T_{i}$ is replaced.

Note that the assertions (3) and (4) will fail if we drop the assumption that $\Lambda$ is hereditary.

We also point out the following trivial but important consequence of Lemma 1.1(4) which we will use frequently.

Corollary 1.2. Let $\Lambda$ be hereditary. If $w: T \rightarrow T^{\prime} \rightarrow T^{\prime \prime}$ is a path in $\overrightarrow{\mathcal{K}}_{\Lambda}$, with $T=M \oplus X$ and $T^{\prime}=M \oplus Y$, then $Y$ is an indecomposable direct summand of $T^{\prime \prime}$.

For a $\Lambda$-module $M$ we denote by fac $M$ the full subcategory of $\bmod \Lambda$ containing the epimorphic images of add $M$. We recall from $\mathrm{H} 2$ the following alternative in the defining property (iii) of a tilting module which will turn out to be important in the next section. 
Proposition 1.3. The following are equivalent for a $\Lambda$-module $M$ with $p d_{\Lambda} M<\infty$ and $\operatorname{Ext}_{\Lambda}^{i}(M, M)=0$ for $i>0$ :

(1) $M$ is tilting module,

(2) $M^{\perp} \subset$ fac $M$.

We recall from U1 the structure of links of dimension one in case $\Lambda$ is hereditary. For this let $M$ be a multiplicity free partial tilting module with $n-2$ non-isomorphic indecomposable direct summands. Let $\overrightarrow{\mathcal{K}}_{\Lambda}(M)$ be the full subquiver of $\overrightarrow{\mathcal{K}}_{\Lambda}$ with vertices $T$ such that $M$ is a direct summand of $T$. Following [U1] and [U2] we call the quiver $\overrightarrow{\mathcal{K}}_{\Lambda}(M)$ a link of dimension one. This result will turn out to be quite useful in the latter sections.

Theorem 1.4. Let $\Lambda$ be hereditary with $n \geq 3$ simple modules and let $M$ be a multiplicity free partial tilting module with $n-2$ non-isomorphic indecomposable direct summands.

(a) If $M$ is faithful and

(1) if $\overrightarrow{\mathcal{K}}_{\Lambda}(M)$ is finite, then $\overrightarrow{\mathcal{K}}_{\Lambda}(M)$ is of the form

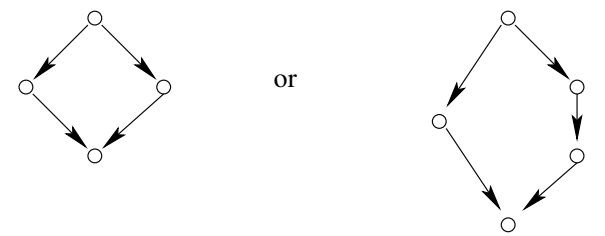

(2) if $\overrightarrow{\mathcal{K}}_{\Lambda}(M)$ is infinite, then $\overrightarrow{\mathcal{K}}_{\Lambda}(M)$ is of the form

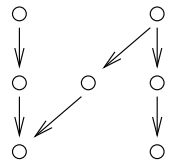

(b) If $M$ is not faithful and

(1) if $\overrightarrow{\mathcal{K}}_{\Lambda}(M)$ is finite, then $\overrightarrow{\mathcal{K}}_{\Lambda}(M)$ is of the form

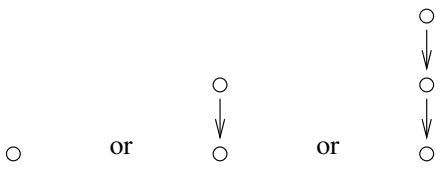


(2) if $\overrightarrow{\mathcal{K}}_{\Lambda}(M)$ is infinite, then $\overrightarrow{\mathcal{K}}_{\Lambda}(M)$ is of the form

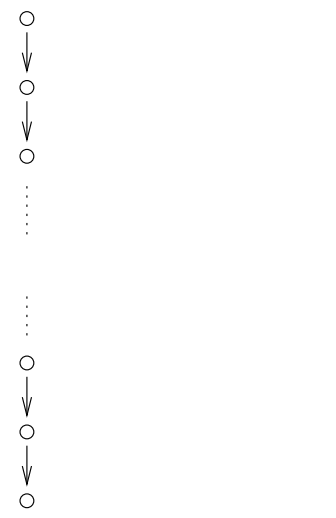

\section{AN INDUCTIVE PROPERTY}

In section 5 we will use orthogonal categories in the sense of [GL (see also [H1]). For the convenience of the reader we recall some of the relevant details.

Although we will use this approach only for hereditary algebras, we will present a more general result which generalizes a result in $\mathrm{AHT}$.

For this let $\Lambda$ be a finite dimensional $k$-algebra over an algebraically closed field $k$. Let $X \in \bmod \Lambda$ with $\operatorname{pd}_{\Lambda} X \leq 1, \operatorname{Ext}_{\Lambda}^{1}(X, X)=0$ and $\operatorname{End}_{\Lambda} X \simeq k$. We define the right orthogonal category $X^{\text {perp }}$ to be the full subcategory of mod $\Lambda$ containing the $\Lambda$-modules $Y$ such that $\operatorname{Hom}_{\Lambda}(X, Y)=0$ and $\operatorname{Ext}_{\Lambda}^{1}(X, Y)=0$. In accordance with [GL] (see also [H1]) we have the following.

Theorem 2.1. The orthogonal category $X^{\text {perp }}$ contains a projective generator $Q$, such that $X^{\text {perp }} \simeq \bmod \Gamma$, where $\Gamma=E n d_{\Lambda} Q$. The number of simple $\Gamma$-modules equals $n-1$. If $\operatorname{Ext}_{\Lambda}^{i}(Q, Q)=0$ for $i \geq 2$, then the following holds:

(1) $\operatorname{Ext}_{\Lambda}^{i}(Y, Z) \simeq \operatorname{Ext}_{\Gamma}^{i}(Y, Z)$ for all $Y, Z \in X^{\text {perp }}$ and all $i \geq 0$.

(2) Let $Z \in X^{\text {perp }}$ with $p d_{\Lambda} Z<\infty$, then $p d_{\Gamma} Z \leq p d_{\Lambda} Z$.

(3) gl.dim $\Gamma \leq$ gl.dim $\Lambda$.

Note that there are examples where $\operatorname{Ext}_{\Lambda}^{i}(Q, Q)=0$ for $i \geq 2$ is not always satisfied (compare for example [H1]).

We also recall from [H1] the following:

Lemma 2.2. Let $S$ be a simple $\Lambda$-module with $p d_{\Lambda} S \leq 1$. Then the projective generator $Q$ of $S^{\text {perp }}$ satisfies $\operatorname{Ext}_{\Lambda}^{i}(Q, Q)=0$ for $i \geq 2$ and $p d_{\Lambda} Q \leq 2$. Moreover for all $Z \in S^{\text {perp }}=\bmod \Gamma$ we have that $p d_{\Lambda} Z \leq p d_{\Gamma} Z+2$.

In subsequent sections we will need the following very useful fact. The first part is well-known. For the convenience of the reader we indicate the argument.

Lemma 2.3. Let $\Lambda$ be a hereditary algebra and $X$ an indecomposable non-projective $\Lambda$-module. Let $P$ be a minimal projective generator of $X^{\text {perp }}$. Then the following hold:

(1) $T^{\alpha}(X)=X \oplus P \in \mathcal{T}_{\Lambda}$.

(2) $\operatorname{Hom}_{\Lambda}\left(T^{\alpha}(X), Y\right) \neq 0$ and $\operatorname{Ext}_{\Lambda}^{1}\left(T^{\alpha}(X), Y\right)=0$ for all modules $Y \in X^{\text {perp }}$. 
(3) If $T^{\prime} \in \mathcal{T}_{\Lambda}$ with $\operatorname{Ext} t_{\Lambda}^{1}\left(X, T^{\prime}\right)=0$, then $T^{\prime} \leq T^{\alpha}(X)$ in $\mathcal{T}_{\Lambda}$.

(4) $T^{\alpha}(X)$ has a unique immediate predecessor in $\overrightarrow{\mathcal{K}}_{\Lambda}$.

(5) $X$ is a direct summand of each immediate successor $T^{\prime}$ of $T^{\alpha}(X)$ in $\overrightarrow{\mathcal{K}}_{\Lambda}$.

Proof. We recall the construction of $P$. Let $s=\operatorname{dim}_{\operatorname{Ext}_{\Lambda}}^{1}\left(X,{ }_{\Lambda} \Lambda\right)$ and let

$$
0 \rightarrow{ }_{\Lambda} \Lambda \rightarrow Q \rightarrow X^{s} \rightarrow 0
$$

be the universal extension. Let $P$ be multiplicity free with add $P=\operatorname{add} Q$. It is well-known that $T^{\alpha}(X)=X \oplus P$ is a tilting module (compare 4.1 in $[\mathrm{R}]$ ). By the construction of the extension $(*)$ we have that the connecting homomorphism $\theta: \operatorname{Hom}_{\Lambda}\left(X, X^{s}\right) \rightarrow \operatorname{Ext}_{\Lambda}^{1}\left(X,{ }_{\Lambda} \Lambda\right)$ is surjective. Since $\operatorname{Hom}_{\Lambda}(X, X)=k$ we see that $\theta$ is an isomorphism. Since $X$ is not projective, $\operatorname{Hom}_{\Lambda}\left(X,{ }_{\Lambda} \Lambda\right)=0$, so we see that $Q \in X^{\text {perp }}$. Thus also $P \in X^{\text {perp }}$. Let $Y \in X^{\text {perp }}$. Applying $\operatorname{Hom}_{\Lambda}(-, Y)$ to $(*)$ shows that $Q$ and thus also $P$ is projective in $X^{\text {perp }}$. It follows from $(*)$ that $Q$ is a generator of $X^{\text {perp }}$, but then $P$ is a minimal projective generator of $X^{\text {perp }}$. Thus $\operatorname{Hom}_{\Lambda}\left(T^{\alpha}(X), Y\right) \neq 0$ and $\operatorname{Ext}_{\Lambda}^{1}\left(T^{\alpha}(X), Y\right)=0$ for all $Y \in X^{\text {perp }}$, which shows (1) and $(2)$.

For (3) let $T^{\prime} \in \mathcal{T}_{\Lambda}$. Applying $\operatorname{Hom}_{\Lambda}\left(-, T^{\prime}\right)$ to the exact sequence $(*)$ and using $\operatorname{Ext}_{\Lambda}^{1}\left(X, T^{\prime}\right)=0$ shows that $\operatorname{Ext}_{\Lambda}^{1}\left(T^{\alpha}(X), T^{\prime}\right)=0$; hence $T^{\prime} \leq T^{\alpha}(X)$ by [HU3.

It follows from $(*)$ that $X$ is generated by $P$. So by Lemma 1.1 we see that $T^{\alpha}(X)$ has an immediate predecessor $T^{\prime \prime}$ in $\overrightarrow{\mathcal{K}}_{\Lambda}$. Let $T^{\prime \prime \prime} \in \mathcal{T}_{\Lambda}$ with $T^{\prime \prime \prime} \neq T^{\prime \prime}$ and $T^{\prime \prime \prime} \rightarrow T^{\alpha}(X)$ in $\overrightarrow{\mathcal{K}}_{\Lambda}$. So $X$ is a direct summand of $T^{\prime \prime \prime}$. Let $T^{\prime \prime \prime}=X \oplus P^{\prime} \oplus Y$ and $T^{\alpha}(X)=X \oplus P^{\prime} \oplus P_{0}$. Since $\operatorname{Hom}_{\Lambda}(X, P)=0$, the sequence connecting the complements $P_{0}$ and $Y$ of $X \oplus P^{\prime}$ implies that $P_{0}$ is generated by $P^{\prime}$; but $P_{0}$ is projective in $X^{\text {perp }}$, a contradiction, thus showing (4).

Let $T^{\alpha}(X) \rightarrow T^{\prime}$ be in $\overrightarrow{\mathcal{K}}_{\Lambda}$. Then $T^{\alpha}(X)=M \oplus Y$ such that $Y$ is cogenerated by $M$. Since $\operatorname{Hom}_{\Lambda}(X, P)=0$ this clearly implies that $X \neq Y$, hence $X$ is a direct summand of $T^{\prime}$, so (5) holds.

We will now study certain subposets of $\mathcal{T}_{\Lambda}$. Let $X$ be a partial tilting module. We define $\mathcal{T}(X)=\left\{T \in \mathcal{T}_{\Lambda} \mid X\right.$ is a direct summand of $\left.T\right\}$.

Lemma 2.4. $\mathcal{T}(X)$ is a convex subposet of $\mathcal{T}_{\Lambda}$.

Proof. For this let $T^{1}, T^{2} \in \mathcal{T}(X)$ and $T \in \mathcal{T}_{\Lambda}$ with $T^{1} \leq T \leq T^{2}$ in $\mathcal{T}_{\Lambda}$. Let $T^{1}=M^{1} \oplus X$ and $T^{2}=M^{2} \oplus X$. By definition $T^{1^{\perp}} \subset T^{\perp}$, so $X \in T^{\perp}$. Also $T^{\perp} \subset T^{2 \perp}$ shows that $\operatorname{Ext}_{\Lambda}^{i}\left(X, T^{\perp}\right)=0$ for $i>0$, hence $X$ is $T^{\perp}-$ projective, or equivalently $X$ is a direct summand of $T$. Thus $T \in \mathcal{T}(X)$.

Corollary 2.5. Let $\overrightarrow{\mathcal{K}}_{\Lambda}(X)$ be the full subquiver of $\overrightarrow{\mathcal{K}}_{\Lambda}$ with vertices in $\mathcal{T}(X)$. Then $\overrightarrow{\mathcal{K}}_{\Lambda}(X)$ is a convex subquiver of $\overrightarrow{\mathcal{K}}_{\Lambda}$.

Now suppose that $X$ is an indecomposable partial tilting module with $\operatorname{pd}_{\Lambda} X \leq 1$. Let $X^{\text {perp }}=\bmod \Gamma$. Note that $\Gamma$ has $n-1$ simple modules. It is easy to construct examples showing that $\mathcal{T}(X)$ is not isomorphic to $\mathcal{T}_{\Gamma}$. In fact, let

$$
\Lambda=k(\circ \rightarrow \circ \rightarrow \cdots \rightarrow \circ \rightarrow \circ)
$$

and let $X$ be the projective-injective indecomposable $\Lambda$-module. Then $\overrightarrow{\mathcal{K}}_{\Lambda}(X)=$ $\overrightarrow{\mathcal{K}}_{\Lambda}$, since $X$ is a direct summand of each tilting module. We will show below that $\mathcal{T}(X)$ is isomorphic to $\mathcal{T}_{\Gamma}$ if $X$ is simple with $\operatorname{id}_{\Lambda} X \leq 1$ and $\operatorname{pd}_{\Lambda} X \leq 1$. Note 
that this applies to the situation investigated in $[\mathrm{AHT}$. We refer to section 5 for a combinatorial description of $\mathcal{T}(X)$ inside $\mathcal{T}_{\Lambda}$ for hereditary algebras.

So we will assume that $S$ is a simple $\Lambda$-module with $\operatorname{pd}_{\Lambda} S \leq 1$ and $\operatorname{id}_{\Lambda} S \leq 1$. Let $S^{\text {perp }}=\bmod \Gamma$. In this situation we will define a map $\varphi_{S}: \mathcal{T}(S) \rightarrow \mathcal{T}_{\Gamma}$. For this let $T \in \mathcal{T}(S)$, so $T=M \oplus S$. Let $f_{1}, \ldots, f_{t}$ be a basis of $\operatorname{Hom}_{\Lambda}(S, M)$. Consider $f: S^{t} \rightarrow M$ with components given by the $f_{i}$. Since $S$ is simple, we have that $f$ is injective. So consider the exact sequence

$$
0 \longrightarrow S^{t} \stackrel{f}{\longrightarrow} M \longrightarrow N \longrightarrow 0 .
$$

By construction we see that $N \in S^{\text {perp }}$. It is easy to see that $N$ is multiplicity free. In fact, if $M=\bigoplus_{i=1}^{n-1} M_{i}$, then $N_{i}=M_{i}$, if $\operatorname{Hom}_{\Lambda}\left(S, M_{i}\right)=0$, and if $\operatorname{Hom}_{\Lambda}\left(S, M_{i}\right) \neq 0$ we have an exact sequence

$$
0 \longrightarrow S^{t_{i}} \stackrel{g_{i}}{\longrightarrow} M_{i} \longrightarrow N_{i} \longrightarrow 0
$$

constructed as $(*)$. Note that we then have that $N=\bigoplus_{i=1}^{n-1} N_{i}$. Let

$$
\psi_{i} \in \operatorname{Hom}_{\Lambda}\left(N_{i}, N_{i}\right) .
$$

Since $\operatorname{Ext}_{\Lambda}^{1}\left(M_{i}, S\right)=0$ we obtain the following commutative diagram of exact sequences:

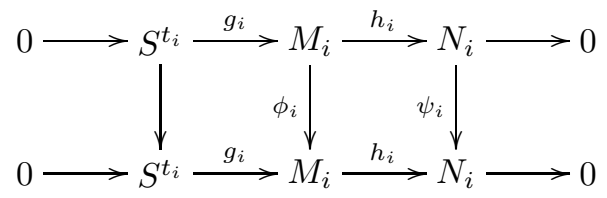

So for each $j \geq 1$ we have that $\phi_{i}^{j} h_{i}=h_{i} \psi_{i}^{j}$. Since $M_{i}$ is indecomposable, or equivalently $\operatorname{End}_{\Lambda} M_{i}$ is local, this clearly implies that $N_{i}$ is indecomposable. Next we show that for $i \neq j$ we have that $N_{i} \not N_{j}$. Assume that $\phi: N_{i} \rightarrow N_{j}$ is an isomorphism. Since $\operatorname{Ext}_{\Lambda}^{1}(M, S)=0$ we obtain the following commutative diagram of exact sequences:

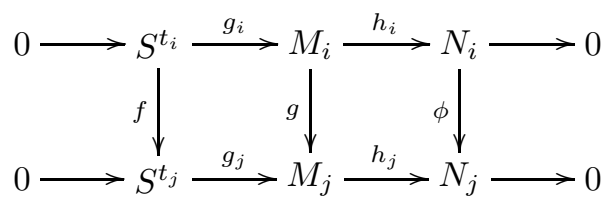

Since the lower sequence does not split, we have that $f \neq 0$, hence $g \neq 0$. Now $\operatorname{ker} g=\operatorname{ker} f \in \operatorname{add} S$ and coker $g=\operatorname{coker} f \in \operatorname{add} S$. So $\operatorname{Ext}_{\Lambda}^{2}(\operatorname{coker} g, \operatorname{ker} g)=0$. But then it follows that $g$ is either mono or epi (compare 4.1 in [HR]). Since $S \oplus M$ is a tilting module, this implies that $g$ is an isomorphism, so $i=j$. Note that the lemma below gives a different proof of the last assertion.

We point out that it is straightforward to check that the isomorphism class of $N$ does not depend on the choice of the basis in $\operatorname{Hom}_{\Lambda}(S, M)$. Indeed, if $f_{1}^{\prime}, \ldots, f_{t}^{\prime} \in$ $\operatorname{Hom}_{\Lambda}(S, M)$ is another basis, there exists an invertible $t \times t$-matrix $\lambda$ over $k$ such that $f^{\prime}=\lambda f$, where $f^{\prime}$ is the map from $S^{t}$ to $M$ with components given by the $f_{i}^{\prime}$. We may consider $\lambda \in \operatorname{Hom}_{\Lambda}\left(S^{t}, S^{t}\right)$. Thus we obtain a commutative diagram of 
exact sequences

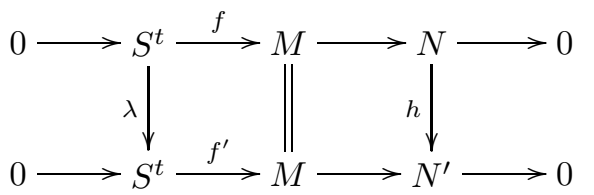

Since $\lambda$ is an isomorphism we infer that $h$ is an isomorphism.

We point out that one could use a functorial approach in this situation. Since we want an explicit description of this correspondence we prefer the approach given here.

Lemma 2.6. $N$ is a $\Gamma$-tilting module.

Proof. Since $\operatorname{pd}_{\Lambda} T<\infty$ then also $\operatorname{pd}_{\Lambda} N<\infty$. By Lemma 2.2 we may apply Theorem 2.1(2). So we have that $\operatorname{pd}_{\Gamma} N<\infty$. Applying $\operatorname{Hom}_{\Lambda}(M,-)$ to (*) shows that $\operatorname{Ext}_{\Lambda}^{i}(M, N)=0$ for $i>0$. Applying $\operatorname{Hom}_{\Lambda}(-, N)$ to $(*)$ and using Theorem 2.1 shows that $\operatorname{Ext}_{\Gamma}^{i}(N, N)=\operatorname{Ext}_{\Lambda}^{i}(N, N)=0$ for $i>0$. Let $X \in N^{\perp} \subset \bmod \Gamma \simeq$ $S^{\text {perp }} \subset \bmod \Lambda$. Applying $\operatorname{Hom}_{\Lambda}(-, X)$ to $(*)$ shows that $X \in T^{\perp}$. Since $T$ is a tilting module we know that $T^{\perp} \subset$ fac $T$, where fac $T$ is the full subcategory of $\bmod \Lambda$ containing the epimorphic images of add $T$. So $X \in \operatorname{fac} T$. Since $X \in S^{\text {perp }}$ it follows that $X \in$ fac $M$. But then $(*)$ shows that $X \in \operatorname{fac} N$, hence $N$ is a $\Gamma$-tilting module by Proposition 1.3 .

So the lemma gives the desired map $\varphi_{S}: \mathcal{T}(S) \rightarrow \mathcal{T}_{\Gamma}$, where $\varphi_{S}(T)=N$ with the notation above.

Theorem 2.7. $\varphi_{S}$ is a bijection of posets.

Proof. We show first that $\varphi_{S}$ is injective. For this let $T^{1}=M^{1} \oplus S$ and $T^{2}=M^{2} \oplus S$ be in $\mathcal{T}(S)$ such that $\varphi_{S}\left(T^{1}\right)=\varphi_{S}\left(T^{2}\right)$. By the definition of $\varphi_{S}$ we have two exact sequences,

$$
0 \rightarrow S^{t_{1}} \rightarrow M^{1} \rightarrow N^{1} \rightarrow 0
$$

and

$$
0 \rightarrow S^{t_{2}} \rightarrow M^{2} \rightarrow N^{2} \rightarrow 0
$$

Since $\varphi_{S}\left(T^{1}\right)=\varphi_{S}\left(T^{2}\right)$ we have add $N^{1}=$ add $N^{2}$. This trivially implies that $\operatorname{Ext}_{\Lambda}^{i}\left(M^{1}, N^{2}\right)=\operatorname{Ext}_{\Lambda}^{i}\left(M^{2}, N^{1}\right)=0$ for $i>0$. Thus also $\operatorname{Ext}_{\Lambda}^{i}\left(M^{1}, M^{2}\right)=0$ and $\operatorname{Ext}_{\Lambda}^{i}\left(M^{2}, M^{1}\right)=0$ for $i>0$, hence $\operatorname{Ext}_{\Lambda}^{i}\left(T^{1} \oplus T^{2}, T^{1} \oplus T^{2}\right)=0$ for $i>0$, in particular $T^{1}=T^{2}$.

Next we show that $\varphi_{S}$ is surjective. For this let $N \in \mathcal{T}_{\Gamma}$ and consider the universal extension

$$
0 \longrightarrow S^{r} \stackrel{g}{\longrightarrow} M \longrightarrow N \longrightarrow 0
$$

so $r=\operatorname{dim} \operatorname{Ext}_{\Lambda}^{1}(N, S)$.

It is easy to see that $M$ is multiplicity free. In fact, let $N=\bigoplus_{i=1}^{n-1} N_{i}$; then $(+)$ is the direct sum of the universal extensions

$(+)_{i} \quad 0 \longrightarrow S^{r_{i}} \longrightarrow M_{i} \longrightarrow N_{i} \longrightarrow 0$

for $1 \leq i \leq n-1$. So $r_{i}=\operatorname{dim} \operatorname{Ext}_{\Lambda}^{1}\left(N_{i}, S\right)$. Thus $M_{i}=N_{i}$ if $r_{i}=0$. If $r_{i} \neq 0$ the exact sequence $(+)_{i}$ is non-split. As above let $\phi_{i} \in \operatorname{Hom}_{\Lambda}\left(M_{i}, M_{i}\right)$. Since 
$\operatorname{Hom}_{\Lambda}\left(S, N_{i}\right)=0$ we obtain the following commutative diagram of exact sequences:

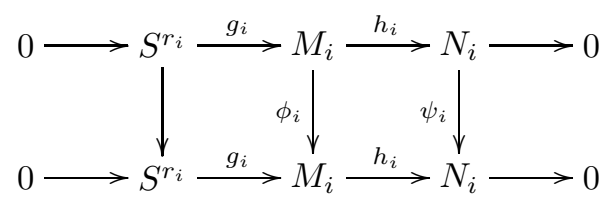

So for each $j \geq 1$ we have that $\phi_{i}^{j} h_{i}=h_{i} \psi_{i}^{j}$. Since $N_{i}$ is indecomposable, or equivalently $\operatorname{End}_{\Lambda} N_{i}$ is local, this clearly implies that $M_{i}$ is indecomposable. Next we show that for $i \neq j$ we have that $M_{i} \not M_{j}$. Assume that $\phi: M_{i} \rightarrow M_{j}$ is an isomorphism. Since $\operatorname{Hom}_{\Lambda}(S, N)=0$ we obtain the following commutative diagram of exact sequences:

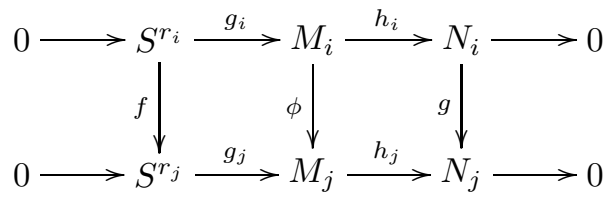

So $g$ is epi, $f$ is mono and cok $f=\operatorname{ker} g$. Now cok $f \in \operatorname{add} S$ and $\operatorname{Hom}_{\Lambda}(S, N)=0$ implies that both $f$ and $g$ are isomorphisms, so $i=j$.

We will now show that $T=M \oplus S$ is a $\Lambda$-tilting module. Since $\operatorname{pd}_{\Gamma} N<\infty$ we have by Lemma 2.2 that also $\operatorname{pd}_{\Lambda} N<\infty$. Since $\operatorname{pd}_{\Lambda} S \leq 1$ we conclude from $(+)$ that $\operatorname{pd}_{\Lambda} T<\infty$. Applying $\operatorname{Hom}_{\Lambda}(S,-)$ to $(+)$ shows that $\operatorname{Ext}_{\Lambda}^{i}(S, M)=0$ for $i>0$. Applying $\operatorname{Hom}_{\Lambda}(-, S)$ to $(+)$ and using the universality of $(+)$ together with $\operatorname{id}_{\Lambda} S \leq 1$ shows that $\operatorname{Ext}_{\Lambda}^{i}(M, S)=0$ for $i>0$. Applying $\operatorname{Hom}_{\Lambda}(-, N)$ to $(+)$ and using the fact that $0=\operatorname{Ext}_{\Gamma}^{i}(N, N)=\operatorname{Ext}_{\Lambda}^{i}(N, N)$ for $i>0$ also shows that $\operatorname{Ext}_{\Lambda}^{i}(M, N)=0$ for $i>0$. Finally applying $\operatorname{Hom}_{\Lambda}(M,-)$ to $(+)$ shows that $\operatorname{Ext}_{\Lambda}^{i}(M, M)=0$ for $i>0$, hence $\operatorname{Ext}_{\Lambda}^{i}(M \oplus S, M \oplus S)=0$ for $i>0$. Next let $X \in(M \oplus S)^{\perp}$ and consider

$$
(++) \quad 0 \longrightarrow S^{m} \stackrel{\alpha}{\longrightarrow} X \stackrel{\beta}{\longrightarrow} Y \longrightarrow 0
$$

as in $(*)$, so $Y \in S^{\text {perp }}$ and also $Y \in(M \oplus S)^{\perp}$. Applying $\operatorname{Hom}_{\Lambda}(-, Y)$ to $(+)$ then shows that $Y \in N^{\perp}$ and thus $Y \in \operatorname{fac} N$, since $N$ is a $\Gamma$-tilting module. So there is $\pi: \tilde{N} \rightarrow Y$ surjective with $\tilde{N} \in$ add $N$. Since $N$ is generated by $M$ there is also $\tilde{\pi}: \tilde{M} \rightarrow Y$ surjective with $\tilde{M} \in \operatorname{add} M$. Since $\operatorname{Ext}_{\Lambda}^{1}\left(\tilde{M}, S^{r}\right)=0$, we obtain $\gamma: \tilde{M} \rightarrow X$ such that $\gamma \beta=\tilde{\pi}$. In particular we obtain the following commutative diagram of exact sequences:

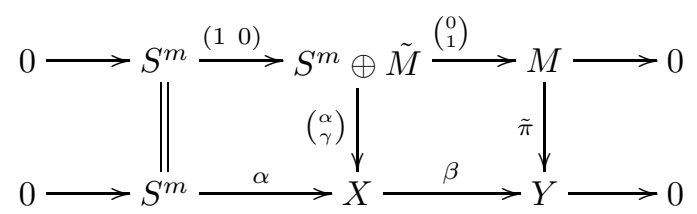

Since the outer maps are surjective, so is $\left(\begin{array}{l}\alpha \\ \gamma\end{array}\right)$; thus $X \in \operatorname{fac}(M \oplus S)$, so $T=M \oplus S$ is a $\Lambda$-tilting module by Proposition 1.3 .

We have shown above that $M$ is multiplicity free, and clearly we have that $\operatorname{dim} \operatorname{Hom}_{\Lambda}(S, M)=r$. Applying $\operatorname{Hom}_{\Lambda}(S,-)$ to $(+)$ shows that $\psi: \operatorname{Hom}_{\Lambda}\left(S, S^{r}\right) \rightarrow$ $\operatorname{Hom}(S, M)$ is an isomorphism. Let $g_{1}, \ldots, g_{r}$ be the components of $g$. Then for 
$\lambda=\left(\lambda_{1}, \ldots, \lambda_{r}\right) \in \operatorname{Hom}_{\Lambda}\left(S, S^{r}\right) \simeq k^{r}$ we obtain that $\psi(\lambda)=\sum_{i=1}^{r} \lambda_{i} g_{i}$. So if $0=\sum_{i=1}^{r} \lambda_{i} g_{i}=\psi(\lambda)$, we have $\lambda=0$, since $\psi$ is an isomorphism. Hence $g_{1}, \ldots, g_{r}$ are linearly independent in $\operatorname{Hom}_{\Lambda}(S, M)$, and so form a basis of $\operatorname{Hom}_{\Lambda}(S, M)$, since $r=\operatorname{dim} \operatorname{Hom}_{\Lambda}(S, M)$. Thus $\varphi_{S}(T)=N$, hence $\varphi_{S}$ is surjective.

It remains to be seen that both $\varphi_{S}$ and $\varphi_{S}^{-1}$ are maps of posets.

For this let $T^{1}=M^{1} \oplus S$ and $T^{2}=M^{2} \oplus S$ be in $\mathcal{T}(S)$ with $T^{1} \leq T^{2}$ in $\mathcal{T}_{\Lambda}$. By definition of $\varphi_{S}$ we have two exact sequences,

$$
0 \longrightarrow S^{t_{1}} \longrightarrow M^{1} \longrightarrow N^{1} \longrightarrow 0
$$

and

$(--)$

$$
0 \longrightarrow S^{t_{2}} \longrightarrow M^{2} \longrightarrow N^{2} \longrightarrow 0 .
$$

So $\varphi_{S}\left(T^{1}\right)=N^{1}$ and $\varphi_{S}\left(T^{2}\right)=N^{2}$. We claim that $N^{1} \leq N^{2}$ in $\mathcal{T}_{\Gamma}$. Let $X \in$ $N^{1}{ }^{\perp}$. Applying $\operatorname{Hom}_{\Lambda}(-, X)$ to $(-)$ shows that $X \in T^{1^{\perp}}$, so $X \in T^{2 \perp}$. Applying $\operatorname{Hom}_{\Lambda}(-, X)$ to $(--)$ and using the fact that $X \in S^{\text {perp }}$ shows that $X \in N^{2}{ }^{\perp}$; thus $\varphi_{S}$ is a map of posets.

Next we investigate $\varphi_{S}^{-1}$. For this let $N^{1}, N^{2} \in \mathcal{T}_{\Gamma}$ with $N^{1} \leq N^{2}$. As in the proof of the surjectivity we obtain two exact sequences:

$$
0 \longrightarrow S^{t_{1}} \longrightarrow M^{1} \longrightarrow N^{1} \longrightarrow 0
$$

and

$$
0 \longrightarrow S^{t_{2}} \longrightarrow M^{2} \longrightarrow N^{2} \longrightarrow 0 .
$$

So $\varphi_{S}^{-1}\left(N^{1}\right)=M^{1} \oplus S$ and $\varphi_{S}^{-1}\left(N^{2}\right)=M^{2} \oplus S$. Let $X \in\left(M^{1} \oplus S\right)^{\perp}$ and consider

$$
(++) \quad 0 \longrightarrow S^{r} \stackrel{\alpha}{\longrightarrow} X \stackrel{\beta}{\longrightarrow} Y \longrightarrow 0
$$

as in (*), so $Y \in S^{\text {perp }}$ and also $Y \in M^{1 \perp}$. Thus $Y \in N^{1}{ }^{\perp}$ by applying $\operatorname{Hom}_{\Lambda}(-, Y)$ to (1). Thus $Y \in N^{2}{ }^{\perp}$, since $N^{1} \leq N^{2}$ in $\mathcal{T}_{\Gamma}$. Applying $\operatorname{Hom}_{\Lambda}(-, Y)$ to $(2)$ then shows that $Y \in\left(M^{2} \oplus S\right)^{\perp}$. But then also $X \in\left(M^{2} \oplus S\right)^{\perp}$, since $\left(M^{2} \oplus S\right)^{\perp}$ is closed under extensions. Thus $T^{1}=M^{1} \oplus S \leq T^{2}=M^{2} \oplus S$ in $\mathcal{T}_{\Lambda}$, which finishes the proof of the theorem.

Corollary 2.8. $\varphi_{S}$ induces an isomorphism $\overrightarrow{\mathcal{K}}_{\Lambda}(S) \rightarrow \overrightarrow{\mathcal{K}}_{\Gamma}$.

Proof. Clearly it is enough to show that minimal inclusions are sent to minimal inclusions under $\varphi_{S}$. So let $T^{1} \rightarrow T^{2}$ be an arrow in $\overrightarrow{\mathcal{K}}_{\Lambda}(S)$. Let $\varphi_{S}\left(T^{2}\right) \leq T \leq$ $\varphi_{S}\left(T^{1}\right)$ for some $T \in \mathcal{T}_{\Gamma}$. Let $\varphi_{S}^{-1}$ be the inverse to $\varphi_{S}$. By the previous theorem $\varphi_{S}^{-1}$ is a map of posets. So $T^{2} \leq \varphi_{S}^{-1}(T) \leq T^{1}$ shows the assertion.

We refer to Corollary $\left[5.9\right.$ for an alternative description of $\overrightarrow{\mathcal{K}}_{\Lambda}(S)$ for a hereditary algebra $\Lambda$ and $S$ a simple $\Lambda$-module.

Note that in the proof of Theorem 2.7 the assumption $\operatorname{id}_{\Lambda} S \leq 1$ is only used to get the surjectivity of $\varphi_{S}$. So in general we just obtain an embedding $\varphi_{S}: \mathcal{T}(S) \rightarrow \mathcal{T}_{\Gamma}$ of posets. The following example shows that if we drop this assumption the map $\varphi_{S}$ need not be surjective. Indeed let $\Lambda$ be given over a field $k$ by the following quiver

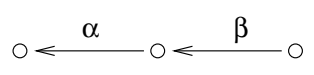


with relation $\beta \alpha=0$. Let $S$ be the unique simple projective $\Lambda$-module. Then $\operatorname{id}_{\Lambda} S=2$. Also ${ }_{\Lambda} \Lambda$ is the unique $\Lambda$-tilting module which has $S$ as a direct summand. So $\mathcal{T}(S)=\circ$. Now $S^{\text {perp }}=\bmod k(\circ \rightarrow \circ)=\bmod \Gamma$. So $\mathcal{T}_{\Gamma}$ contains two elements. The map $\varphi_{S}$ sends ${ }_{\Lambda} \Lambda$ to ${ }_{\Gamma} \Gamma$.

We point out that if $S$ is simple injective and $\operatorname{pd}_{\Lambda} S \leq 1$, we are in the situation investigated in $\mathrm{AHT}$.

Trivially, if $\Lambda$ is hereditary, then each simple $\Lambda$-module $S$ satisfies our assumptions. However there are even tilted algebras with no simple $\Lambda$-module $S$ satisfying these assumptions. In fact, let $\Lambda$ be given over a field $k$ by the following quiver with relations:

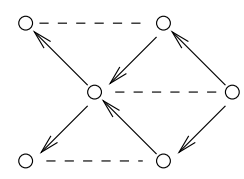

The following properties are easily verified. $\Lambda$ is a tilted algebra of type $\mathbb{E}_{6}$ and there is no simple $\Lambda$-module $S$ with both $\operatorname{pd}_{\Lambda} S \leq 1$ and $\operatorname{id}_{\Lambda} S \leq 1$.

We also point out that for $\Lambda_{n}=k(\circ \rightarrow \circ \cdots \circ \rightarrow \circ)$ with $n$ vertices each tilting module $T$ has a simple direct summand $S$. So in this case $\overrightarrow{\mathcal{K}}_{\Lambda}=\bigcup_{S} \overrightarrow{\mathcal{K}}_{\Gamma(S)}$, where $\bmod \Gamma(S)=S^{\text {perp }}$. Note that $\Gamma(S) \simeq \Lambda_{n-1}$ for each $S$, but the embeddings of $S^{\text {perp }}$ into $\bmod \Lambda_{n}$ vary for the different choices of $S$. The union above is clearly not a disjoint union.

We also give the following example which shows that there is $\overrightarrow{\mathcal{K}}_{\Gamma} \subset \overrightarrow{\mathcal{K}}_{\Lambda}$ for $\bmod \Gamma \simeq S^{\text {perp }}$ and $S$ a simple $\Lambda$-module such that $\overrightarrow{\mathcal{K}}_{\Gamma}$ is not connected while $\overrightarrow{\mathcal{K}}_{\Lambda}$ is connected. Indeed, consider the following two quivers $\vec{\Delta}$ and $\vec{\Delta}$
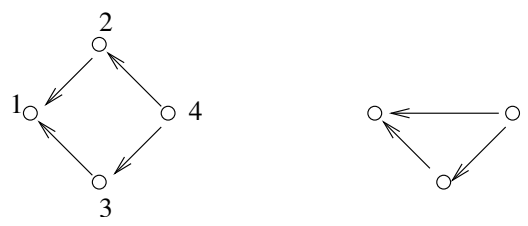

Let $\Lambda=k \vec{\Delta}$ and let $S=S_{2}$ be the simple $\Lambda$-module associated with the vertex of $\vec{\Delta}$ labelled 2 . Then $S^{\text {perp }} \simeq \bmod \Gamma$ for $\Gamma=k \vec{\Delta}^{\prime}$. For this note that the projective generator ${ }_{\Lambda} Q$ of $S^{\text {perp }}$ is given by

$$
{ }_{\Lambda} Q={ }_{\Lambda} P(2) \oplus \tau_{\Lambda}^{-} P(1) \oplus \tau_{\Lambda}^{-} P(2) .
$$

It is well-known that $\overrightarrow{\mathcal{K}}_{\Lambda}$ is connected and $\overrightarrow{\mathcal{K}}_{\Gamma}$ has exactly two connected components U2].

We will also need orthogonal categories for $\Lambda$-modules which may be decomposable. For this let $\Lambda$ be a hereditary algebra and let $M$ be a $\Lambda$-module with $\operatorname{Ext}_{\Lambda}^{1}(M, M)=0$. We define the right orthogonal category $M^{\text {perp }}$ to be the full subcategory of $\bmod \Lambda$ containing the $\Lambda$-modules $X$ such that $\operatorname{Hom}_{\Lambda}(M, X)=0$ and $\operatorname{Ext}_{\Lambda}^{1}(M, X)=0$. Note that the indecomposable direct summands $M_{i}$ of $M$ can be ordered such that $\operatorname{Hom}_{\Lambda}\left(M_{i}, M_{j}\right)=0$ if $i>j$, so this definition is compatible with the definition given above. We assume that $M$ has $t$ non-isomorphic indecomposable direct summands. Then it is shown in $\mathrm{GL}$ that $M^{\text {perp }} \simeq \bmod \Gamma$, where 
$\Gamma$ is a finite dimensional hereditary algebra with $n-t$ simple modules. Moreover the inclusion $M^{\text {perp }} \rightarrow \bmod \Lambda$ is an exact embedding. One situation will be of particular importance for us. Let $M$ be a faithful projective $\Lambda$-module with $n-2$ non-isomorphic indecomposable summands, say $M=\bigoplus_{i=1}^{n-2} P(i)$. We will assume that $n \geq 3$. Clearly $\bmod \Gamma=M^{\text {perp }}$ is equivalent to the subcategory of $\Lambda$-modules with composition factors of the form $S(n-1)$ and $S(n)$. Let $T=M \oplus X \oplus Y$ be a tilting module such that $X$ and $Y$ are not projective. Then by the AuslanderReiten formula (compare for example 2.4 in $\left[\underline{\mathbb{R}}\right.$ ) we see that $\tau_{\Lambda}(X \oplus Y) \in M^{\text {perp }}$ is a $\Gamma$ - tilting module. Moreover we obtain an embedding of quivers $\overrightarrow{\mathcal{K}}_{\Gamma} \rightarrow \overrightarrow{\mathcal{K}}_{\Lambda}$ such that the unique sink $D \Gamma_{\Gamma}$ of $\overrightarrow{\mathcal{K}}_{\Gamma}$ is mapped to the unique sink of $\overrightarrow{\mathcal{K}}_{\Lambda}(M)$. In this way Theorem 1.4 can be interpreted such that the first case corresponds to the case where $\Gamma$ is a product of two copies of $k$, that the second case corresponds to the case where $\Gamma$ is of type $\mathbb{A}_{2}$, and the last case corresponds to the case where $\Gamma$ is isomorphic to a representation infinite hereditary algebra with two simple modules. Of course there are infinitely many such examples.

\section{Detection of Simples}

In the introduction we have defined the partially ordered set $\left(\mathcal{T}_{\Lambda}, \leq\right)$ and the quiver $\overrightarrow{\mathcal{K}}_{\Lambda}$ of tilting modules. Since we are mainly interested in the structure of $\overrightarrow{\mathcal{K}}_{\Lambda}$ for hereditary algebras, we will assume in this section that $\Lambda$ is a finite dimensional hereditary $k$-algebra for an algebraically closed field $k$. Thus $\Lambda \simeq k \vec{\Delta}$, where $k \vec{\Delta}$ denotes the path algebra over $k$ of a finite quiver without oriented cycles. We denote by $n$ the number of vertices of $\vec{\Delta}$. The main aim of this section is to determine this number $n$ from $\overrightarrow{\mathcal{K}}_{\Lambda}$ in case $\Lambda$ is connected. A more general result will be shown in section 5 .

Let $T \in \overrightarrow{\mathcal{K}}_{\Lambda}$. We denote by $s(T)$ (resp. $e(T)$ ) the number of arrows starting (resp. ending) at $T$ in $\overrightarrow{\mathcal{K}}_{\Lambda}$.

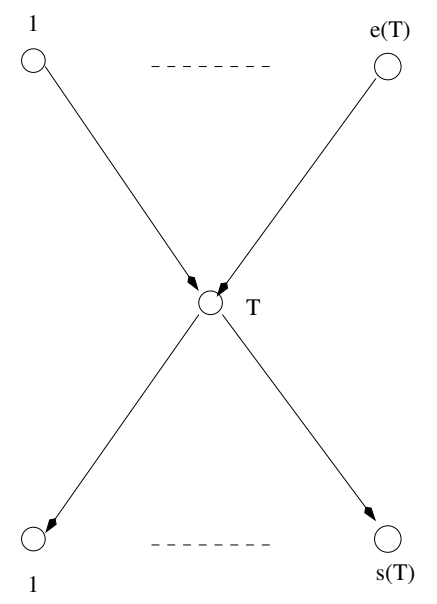

Denote by $K_{0}(\Lambda)$ the Grothendieck group of $\Lambda$. We recall from HU2].

Lemma 3.1. $\delta(T)=s(T)+e(T) \leq r k K_{0}(\Lambda)=n$.

We say that $T$ is saturated if equality holds. Note that the proof of Lemma 3.1 shows that $T$ is saturated if and only if $T[i]$ is faithful or equivalently sincere for 
each $1 \leq i \leq n$. Here we will investigate the situation more closely. We denote it by $\delta(\Lambda)=\max \delta(\mathrm{T})$. So $\delta(\Lambda)$ is the degree of $\overrightarrow{\mathcal{K}}_{\Lambda}$.

For a $\Lambda$-module $X$ we denote by $\operatorname{dim} X \in K_{0}(\Lambda)$ its dimension vector which we will identify with the corresponding element in $\mathbb{Z}^{n}$. The following is an easy generalization of 3.2 in HU2. We also refer to HU2 for explicit ways to compute $e(T)$ and $s(T)$ independently.

Proposition 3.2. If $T \in \bmod k \vec{\Delta}$ is a tilting module, then the degree $\delta(T)=$ $n-\left|\left\{i \mid(\operatorname{dim} T)_{i}=1\right\}\right|$.

Proof. We consider the almost complete partial tilting modules $T[1], \ldots, T[n]$. We may assume that $T[1], \ldots, T[r]$ are faithful and that $T[r+1], \ldots, T[n]$ are not faithful. By Lemma 1.1 and the definition of $\overrightarrow{\mathcal{K}}_{\Lambda}$ we clearly have that $\delta(T)=r$.

Let $r+1 \leq j \leq n$, so there is $i_{j}$ such that $\operatorname{Hom}_{\Lambda}\left(T[j], I\left(i_{j}\right)\right)=0$. If $r+1 \leq j^{\prime} \leq n$ and $j \neq j^{\prime}$, then $i_{j} \neq i_{j^{\prime}}$ for add $T=\operatorname{add} T[j] \oplus T\left[j^{\prime}\right]$ and $T$ is faithful.

Let $r+1 \leq j \leq n$. Consider $\Gamma=\operatorname{End}_{\Lambda} T$. By the tilting theory we have that $\operatorname{Hom}_{\Lambda}\left(T, I\left(i_{j}\right)\right)$ is an indecomposable $\Gamma$-module. Set $P_{j}=\operatorname{Hom}_{\Lambda}\left(T, T_{j}\right)$ and $S_{j}=$ top $P_{j}$. Now

$$
\operatorname{Hom}_{\Lambda}\left(T, I\left(i_{j}\right)\right) \simeq S_{j}^{\operatorname{dim} \operatorname{Hom}_{\Lambda}\left(T, I\left(i_{j}\right)\right)} \simeq S_{j}^{\operatorname{dim} \operatorname{Hom}_{\Lambda}\left(T_{j}, I\left(i_{j}\right)\right)} .
$$

$\operatorname{Hom}_{\Lambda}\left(T, I\left(i_{j}\right)\right)$ indecomposable implies that $\operatorname{dim}_{\operatorname{Hom}}\left(T, I\left(i_{j}\right)\right)=1$. So there is $1 \leq i \leq n$ with $(\operatorname{dim} T)_{i}=1$, and by the first remark we see that $\mid\left\{i \mid(\operatorname{dim} T)_{i}=\right.$ $1\} \mid \geq n-r=n-\delta(T)$.

For the other inequality let $1 \leq i \leq n$ with $(\operatorname{dim} T)_{i}=1$. Then there is $1 \leq j_{i} \leq n$ such that $T=T\left[j_{i}\right] \oplus T_{j_{i}}$ with $\left(\operatorname{dim} T_{j_{i}}\right)_{i}=1$. Then $\left(\operatorname{dim} T\left[j_{i}\right]\right)_{i}=0$, so $T\left[j_{i}\right]$ is not faithful, hence $r+1 \leq j_{i} \leq n$. This finishes the proof.

The following is an immediate consequence of Proposition 3.2

Corollary 3.3. Let $\Lambda=k \vec{\Delta}$. Then $s\left({ }_{\Lambda} \Lambda\right)=n-\mid$ sources in $\vec{\Delta} \mid$ and $e\left(D \Lambda_{\Lambda}\right)=$ $n-\mid$ sinks in $\vec{\Delta} \mid$. So $s\left({ }_{\Lambda} \Lambda\right)+e\left(D \Lambda_{\Lambda}\right) \geq n$ with equality if and only if there is no path of length two in $\vec{\Delta}$.

Proof. Clearly we have that $i \in \vec{\Delta}$ is a source if and only if $\left(\operatorname{dim}_{\Lambda} \Lambda\right)_{i}=1$ and $i \in \vec{\Delta}$ is sink if and only if $\left(\operatorname{dim} D \Lambda_{\Lambda}\right)_{i}=1$. The result now follows from Proposition 3.2 .

Next we will show how to deduce the number $n$ of simple $\Lambda$-modules from $\overrightarrow{\mathcal{K}}_{\Lambda}$. Recall that we have denoted by $\delta(\Lambda)$ the degree of $\overrightarrow{\mathcal{K}}_{\Lambda}$.

Proposition 3.4. Let $\Lambda$ be a connected finite dimensional hereditary $k$-algebra. Then $\delta(\Lambda)=n$ if and only if $\Lambda \neq k(\circ \rightarrow \circ \cdots \circ \rightarrow \circ)$ with $n$ vertices. Moreover, if $\Lambda \simeq k(\circ \rightarrow \circ \cdots \circ \rightarrow \circ)$ with $n$ vertices, then $\delta(\Lambda)=s\left({ }_{\Lambda} \Lambda\right)=n-1$.

Proof. If $\Lambda \simeq k(\circ \rightarrow \circ \cdots \circ \rightarrow \circ)$, denote by $P$ the unique indecomposable projectiveinjective $\Lambda$-module. If $T$ is a tilting module, then $P$ is an indecomposable direct summand of $T$, so $T=P \oplus T^{\prime}$. Since $P$ is neither generated nor cogenerated by $T^{\prime}$, we infer that $\delta(T)<n$.

Conversely, assume that $\Lambda \nsucceq k(\circ \rightarrow \circ \cdots \circ \rightarrow \circ)$. If $\Lambda$ is of infinite representation type it is well-known that there is $t \in \mathbb{N} \operatorname{such}\left(\operatorname{dim} \tau_{\Lambda}^{-t} \Lambda\right)_{i} \geq 2$ for all $i$. Here of course we use the fact that $\Lambda$ is connected. So by the proposition we see that $\delta(\Lambda)=$ $n$. If $\Lambda$ is of finite representation type, let $X$ be the indecomposable $\Lambda$-module 
corresponding to the maximal root. Since $\Lambda \neq k(\circ \rightarrow \circ \cdots \circ \rightarrow \circ)$ and $\Lambda$ is connected, we have that $X$ is not both projective and injective. We assume that $X$ is not injective. The other case can be dealt with in a dual way. Consider the Auslander-Reiten sequence $0 \rightarrow X \rightarrow Y \rightarrow \tau^{-} X \rightarrow 0$ starting in $X$. Since $X$ is sincere, we infer that $Y$ is sincere. Clearly $X \oplus Y$ is a partial tilting module, since $\Lambda$ is hereditary. Let $T=X \oplus Y \oplus C$ be a tilting module. Then clearly $(\operatorname{dim} T)_{i} \geq 2$ for all $i$, hence $\delta(\Lambda)=n$.

Now let $\Lambda=k(\circ \rightarrow \circ \cdots \circ \rightarrow \circ)$ with $n$ vertices. We have seen above that $\delta(\Lambda) \leq n-1$. Since there is a unique source in $\Lambda$ we have by Corollary 3.3 that $n-1=s\left({ }_{\Lambda} \Lambda\right)=\delta\left({ }_{\Lambda} \Lambda\right) \leq \delta(\Lambda)$, hence $\delta(\Lambda)=s\left({ }_{\Lambda} \Lambda\right)=n-1$.

Corollary 3.5. Let $\Lambda$ be a connected hereditary algebra. Then $n-1 \leq \delta(\Lambda) \leq n$.

Proof. This follows immediately from the previous proposition.

We summarize these results in the following corollary, which also shows how to detect the number of simple $\Lambda$-modules from $\overrightarrow{\mathcal{K}}_{\Lambda}$.

Corollary 3.6. Let $\Lambda$ be a connected hereditary algebra. If $s\left({ }_{\Lambda} \Lambda\right)=\delta(\Lambda)$, then $n=\delta(\Lambda)+1$. Otherwise $n=\delta(\Lambda)$.

Proof. By Corollary 3.3 we have that $s\left({ }_{\Lambda} \Lambda\right) \leq n-1$, since there is at least one source. So if $s\left({ }_{\Lambda} \Lambda\right)=\delta(\Lambda)$, we infer by Corollary 3.5 that $\delta(\Lambda)=n-1$, so by Proposition 3.4 we have that $\Lambda \simeq k(\circ \rightarrow \circ \cdots \circ \rightarrow \circ)$ with $n$ vertices, hence $n=\delta(\Lambda)+1$. Otherwise we have that $s\left({ }_{\Lambda} \Lambda\right) \neq \delta(\Lambda)$. So we conclude from Proposition 3.4 that $\Lambda \not f k(\circ \rightarrow \circ \cdots \circ \rightarrow \circ)$. Using Proposition 3.4 again we finally see that $n=\delta(\Lambda)$.

We remark that the proof of Proposition 3.4 actually shows that we can read off the degree $\delta(\Lambda)$ by just considering the connected component of $\overrightarrow{\mathcal{K}}_{\Lambda}$ containing ${ }_{\Lambda} \Lambda$.

\section{The Dynkin CASE}

In this section we investigate more closely the situation of representation finite hereditary algebras. We assume that $\Lambda$ is connected. Thus $\Lambda \simeq k \vec{\Delta}$, where $\Delta$ is a Dynkin diagram of the form $\mathbb{A}_{n}, \mathbb{D}_{n}, \mathbb{E}_{6}, \mathbb{E}_{7}$, or $\mathbb{E}_{8}$ (see for example ARS]). In this situation $\overrightarrow{\mathcal{K}}_{\Lambda}$ is finite, so we can read off the number of tilting modules and by the result in the previous section the number of simple modules. In this section we will show how to determine the number of indecomposable $\Lambda$-modules (up to isomorphism). As an easy consequence we will see that this already determines $\Delta$ from $\overrightarrow{\mathcal{K}}_{\Lambda}$. The precise reconstruction (i.e. the orientation) will be done in section 6.

Since $\overrightarrow{\mathcal{K}}_{\Lambda}$ is finite, we may define $\ell\left(\overrightarrow{\mathcal{K}}_{\Lambda}\right)=\max \left\{l(w) \mid w\right.$ path in $\left.\overrightarrow{\mathcal{K}}_{\Lambda}\right\}$, where for a path $w \in \overrightarrow{\mathcal{K}}_{\Lambda}$ we denote by $l(w)$ the number of arrows occurring in $w$. By $m(\Delta)$ we denote the number of positive roots in the root system associated with $\Delta$. So $m(\Delta)$ coincides with the number of isomorphism classes of indecomposable $k \vec{\Delta}$-modules.

The following theorem is an easy application of the representation theory of Dynkin quivers, but it still seems to be worthwhile mentioning.

Theorem 4.1. $\ell\left(\overrightarrow{\mathcal{K}}_{\Lambda}\right)=m(\Delta)-n$.

Proof. Let $\Lambda=k \vec{\Delta}$ for a Dynkin diagram $\Delta$. If $T \rightarrow T^{\prime}$ is an arrow in $\overrightarrow{\mathcal{K}}_{\Lambda}$ we have that $T^{\perp} \subset T^{\perp}$. Let $T=M \oplus X$ and $T^{\prime}=M \oplus Y$. Then the non-split 
exact sequence connecting the complements (compare Lemma 1.1) shows that the inclusion is proper. In fact, $X \in T^{\perp}$, but $X \notin T^{\perp}$. We denote by $m(T)$ the number of indecomposable $\Lambda$-modules (up to isomorphism) in $T^{\perp}$. Since $D \Lambda_{\Lambda} \in T^{\perp}$ for all $T$, we clearly have that $m(\Delta)=m\left({ }_{\Lambda} \Lambda\right) \geq m(T) \geq m\left(D \Lambda_{\Lambda}\right)=n$ for all $T \in \overrightarrow{\mathcal{K}}_{\Lambda}$. Thus $\ell\left(\overrightarrow{\mathcal{K}}_{\Lambda}\right) \leq m(\Delta)-n$.

For the converse inequality we will now construct a path in $\overrightarrow{\mathcal{K}}_{\Lambda}$ of the form

$$
T_{0}={ }_{\Lambda} \Lambda \rightarrow T_{1} \rightarrow \cdots \rightarrow T_{r-1} \rightarrow T_{r}=D \Lambda_{\Lambda}
$$

such that $m\left(T_{i}\right)=m\left(T_{i+1}\right)+1$ for each $0 \leq i \leq r-1$, and for each $0 \leq i \leq r$ we have that End $T_{i}$ is hereditary. We assume that $T_{i} \neq D \Lambda_{\Lambda}$ is constructed and show how to construct $T_{i+1}$ with the desired properties. Since $T_{i} \neq D \Lambda_{\Lambda}$, there is an indecomposable direct summand $X$ of $T_{i}=M \oplus X$ such that $\operatorname{Hom}_{\Lambda}(M, X)=0$ and $X$ is not injective. Since End $T_{i}$ is hereditary we know that the indecomposable direct summands of $T_{i}$ form a complete slice (compare 4.2 in $[\underline{\mathrm{R}}]$ ). Let $0 \rightarrow X \rightarrow$ $E \rightarrow \tau^{-} X \rightarrow 0$ be the Auslander-Reiten sequence starting in $X$. Then $E$ is a direct summand of $T_{i}$. Set $T_{i+1}=M \oplus \tau^{-} X$. It is straightforward to check that $T_{i+1}$ is a tilting module such that $m\left(T_{i}\right)=m\left(T_{i+1}\right)+1$ and that End $T_{i+1}$ is hereditary. Since $\overrightarrow{\mathcal{K}}_{\Lambda}$ is finite this process has to stop, and this is precisely the case when we reach $D \Lambda_{\Lambda}$, which finishes the proof of the theorem.

The following is an immediate consequence of the theorem above.

Corollary 4.2. Let $\vec{\Delta}$ be a Dynkin quiver and $\Lambda=k \vec{\Delta}$. Then $\ell\left(\overrightarrow{\mathcal{K}}_{\Lambda}\right)$ is independent of the orientation.

We note, however, that a corresponding result for the length of a shortest path from the source to the sink of $\overrightarrow{\mathcal{K}}_{\Lambda}$ will fail, as the following example shows. Let $\Lambda=k(\circ \rightarrow \circ \rightarrow \circ)$ and $\Lambda^{\prime}=k(\circ \rightarrow \circ \leftarrow \circ)$. In the first case a shortest path from the source to the sink of $\overrightarrow{\mathcal{K}}_{\Lambda}$ will have length two, while in the second case it will have length three, which can be seen from the quivers $\overrightarrow{\mathcal{K}}_{\Lambda}$ and $\overrightarrow{\mathcal{K}}_{\Lambda^{\prime}}$ depicted below.
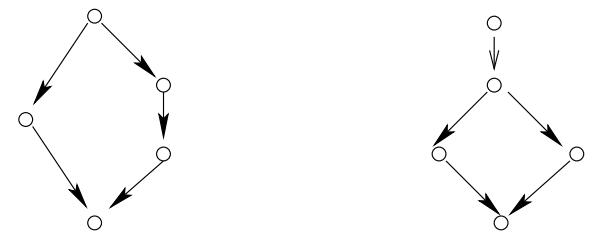

In the following corollary we characterize the tilting modules lying on maximal paths in $\overrightarrow{\mathcal{K}}_{\Lambda}$.

Corollary 4.3. Let $T$ be a vertex of $\overrightarrow{\mathcal{K}}_{\Lambda}$. The indecomposable direct summands of $T$ form a complete slice if and only if $T$ lies on a path of maximal length. In particular, the number of complete slices coincides with the number of different vertices on paths of maximal length.

Proof. If the indecomposable direct summands of $T$ form a complete slice, we may construct as in the proof of the theorem a path from $T$ to $D \Lambda_{\Lambda}$ and dually a path from ${ }_{\Lambda} \Lambda$ to $T$. The concatenation of these two paths yields a path of maximal length. 
Conversely, let $T$ be a vertex on path $w$ of maximal length. As pointed out above this path has to start in ${ }_{\Lambda} \Lambda$ and terminate in $D \Lambda_{\Lambda}$. Moreover we have for each arrow $T \rightarrow T^{\prime}$ on $w$ that $m(T)=m\left(T^{\prime}\right)+1$. We may assume that the indecomposable direct summands of $T$ form a complete slice, and we will show that $T^{\prime}$ is of the form constructed as in the proof of the theorem. Since $T \rightarrow T^{\prime}$ in $\overrightarrow{\mathcal{K}}_{\Lambda}$ we have that $T=M \oplus X$ and that $T^{\prime}=M \oplus Y$. Since $m(T)=m\left(T^{\prime}\right)+1$, we have that $X$ is the unique indecomposable module in $T^{\perp}$ not contained in $T^{\prime \perp}$. Since $X$ is cogenerated by $M$ we have by Lemma 1.1(3) that $X$ is not generated by $M$. Since $Y$ is generated by $M$ this shows that $X$ is not generated by $T^{\prime}$. If $\operatorname{Hom}(M, X) \neq 0$ we have by tilting theory a non-split exact sequence $0 \rightarrow t(X) \rightarrow X \rightarrow X / t(X) \rightarrow 0$ with $t(X) \in T^{\perp}$. Clearly, $X / t(X) \in T^{\perp}$ and $X / t(X) \notin T^{\perp}$. Since by assumption $t(X) \neq 0$, there is an indecomposable direct summand $Z$ of $X / t(X)$ with $Z \in T^{\perp}$ and $Z \notin T^{\prime \perp}$. Since $Z \neq X$ this gives a contradiction to $m(T)=m\left(T^{\prime}\right)+1$. Thus $\operatorname{Hom}(M, X)=0$. Trivially $X$ is not injective, as the non-split exact sequence connecting the complements induced by the arrow $T \rightarrow T^{\prime}$ in $\overrightarrow{\mathcal{K}}_{\Lambda}$ shows. Since the indecomposable direct summands of $T$ form a complete slice, we conclude that the exact sequence connecting the complements is the Auslander-Reiten sequence starting in $X$; thus $T^{\prime}=M \oplus \tau^{-} X$ and $\operatorname{End}_{\Lambda} T^{\prime}$ is hereditary.

It is well-known that $m\left(\mathbb{A}_{n}\right)=\frac{n(n+1)}{2}, m\left(\mathbb{D}_{n}\right)=n(n-1), m\left(\mathbb{E}_{6}\right)=36, m\left(\mathbb{E}_{7}\right)=$ 63 and $m\left(\mathbb{E}_{8}\right)=120$. It is easily checked that for a fixed $n$ these numbers are pairwise different. By Corollary 3.6 we can compute $n$ from $\overrightarrow{\mathcal{K}}_{\Lambda}$, and thus by Theorem 4.1 we can compute $m(\Delta)$ from $\overrightarrow{\mathcal{K}}_{\Lambda}$; hence we have the following corollary.

Corollary 4.4. If $\overrightarrow{\mathcal{K}}_{\Lambda}$ is finite, then $\Delta$ is uniquely determined (up to isomorphism) by $\overrightarrow{\mathcal{K}}_{\Lambda}$.

A different way of determining $m(\Delta)$ is given in the following proposition.

Proposition 4.5. $m(\Delta)=\left|\left\{T \in \mathcal{T}_{\Lambda} \mid e(T)=1\right\}\right|+n$.

Proof. Let $\vec{\Delta}$ be a Dynkin quiver. Clearly it is enough to construct a bijection between the isomorphism classes of indecomposable non-projective $k \vec{\Delta}$-modules and the set $\left\{T \in \mathcal{T}_{\Lambda} \mid e(T)=1\right\}$. Let $X$ be an indecomposable non-projective $k \vec{\Delta}$-module. We consider the category $X^{\text {perp }}$. Let $P \in X^{\text {perp }}$ be a minimal projective generator. By Lemma 2.3 we have that $T=X \oplus P$ is a tilting module and that $e(T)=1$. This defines a map between the two sets above which is injective, since for $X \not X^{\prime}$ indecomposable and non-projective $X \oplus P=X^{\prime} \oplus P^{\prime}$ implies that $X$ is a direct summand of $P^{\prime}$, so in $X^{\prime \text { perp }}$ and that $X^{\prime}$ is a direct of summand of $P$. By 4.1 in $\left[\mathrm{R}\right.$ each indecomposable direct summand $P_{i}$ of $P$ satisfies $\operatorname{Hom}_{\Lambda}\left(P_{i}, X\right) \neq 0$, a contradiction. So the map defined above is injective. Next we show that it is also surjective. For this let $T \in \mathcal{T}_{\Lambda}$ with $e(T)=1$. Let $T=M \oplus X$, where $X$ is the unique indecomposable direct summand of $T$ which gives rise to the arrow in $\overrightarrow{\mathcal{K}}_{\Lambda}$ to $T$. We claim that $M \in X^{\text {perp }}$ is projective. If $\operatorname{Hom}_{\Lambda}(X, M) \neq 0$, there is an indecomposable direct summand $M_{i}$ of $M$ and $0 \neq f_{i}: X \rightarrow M_{i}$. Now $f_{i}$ is either mono or epi $\mathrm{HR}$. Since $X$ is generated by $M$ it is not cogenerated by $M$, so $f_{i}$ is not mono, and since $e(T)=1$ we have that $f_{i}$ is not epi. So $M \in X^{\text {perp }}$. Clearly, $M$ is a tilting module in $X^{\text {perp }}$. If $M$ is not projective in $X^{\text {perp }}$, there is an indecomposable direct summand $Y$ of $M=N \oplus Y$ such that $Y$ is generated by $N$. But then $Y$ is also generated by $N \oplus X$, in contrast to $e(T)=1$. 


\section{Tilting MOdules ASSOCIATED WITH SIMPlE MODUlES}

In this section we will investigate more closely the neighbors of ${ }_{\Lambda} \Lambda$ and $D \Lambda_{\Lambda}$ in $\overrightarrow{\mathcal{K}}_{\Lambda}$. First we introduce tilting modules associated with certain simple modules.

Lemma 5.1. Let $\Lambda=k \vec{\Delta}$ be a hereditary algebra. Let $S_{i}$ be a simple $\Lambda$-module which is not injective. Then $T_{S_{i}}={ }_{\Lambda} \Lambda[i] \oplus \tau_{\Lambda}^{-} S_{i}$ is a tilting module. In this way we obtain all immediate successors of $\Lambda \Lambda$ in $\overrightarrow{\mathcal{K}}_{\Lambda}$.

Proof. Since $\Lambda$ is hereditary and $T_{S_{i}}$ has the right number of non-isomorphic indecomposable direct summands, it is enough to show that $\operatorname{Ext}_{\Lambda}^{1}\left(T_{S_{i}}, T_{S_{i}}\right)=0$. In fact, apply the Auslander-Reiten formula (compare for example 2.4 in $[\mathbf{R}]$ ) to obtain the following chain of isomorphisms:

$$
\begin{aligned}
\operatorname{Ext}_{\Lambda}^{1}\left(T_{S_{i}}, T_{S_{i}}\right) & \simeq D \operatorname{Hom}_{\Lambda}\left(T_{S_{i}}, \tau_{\Lambda} T_{S_{i}}\right)=D \operatorname{Hom}_{\Lambda}\left({ }_{\Lambda} \Lambda[i] \oplus \tau_{\Lambda}^{-} S_{i}, S_{i}\right) \\
& =D \operatorname{Hom}_{\Lambda}\left(\tau_{\Lambda}^{-} S_{i}, S_{i}\right) \simeq \operatorname{Ext}_{\Lambda}^{1}\left(S_{i}, S_{i}\right)=0 .
\end{aligned}
$$

Thus $T_{S_{i}}$ is a tilting module.

By Corollary 3.3 we obtain in this way all immediate successors of ${ }_{\Lambda} \Lambda$ in $\overrightarrow{\mathcal{K}}_{\Lambda}$.

Dually we obtain for each simple $\Lambda$-module $S_{i}$ which is not projective a tilting module $T^{S_{i}}=\left(D \Lambda_{\Lambda}\right)[i] \oplus \tau S_{i}$ yielding all immediate predecessors of $D \Lambda_{\Lambda}$ in $\overrightarrow{\mathcal{K}}_{\Lambda}$. $\vec{\Delta}$.

Given a quiver $\vec{\Delta}$ we will identify the simple $k \vec{\Delta}$-modules with the vertices of

Lemma 5.2. Let $\Lambda=k \vec{\Delta}$ be a hereditary algebra and let $S, S^{\prime}$ be different simple $\Lambda$-modules which are not injective. Then there is no edge in $\vec{\Delta}$ between $S$ and $S^{\prime}$ if and only if $T_{S}$ and $T_{S^{\prime}}$ are contained in $\overrightarrow{\mathcal{K}}_{\Lambda}$ in a diamond of the form

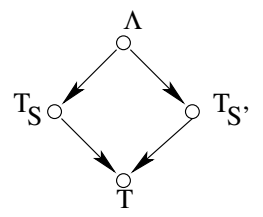

Proof. Let $S=S_{i}$ and $S^{\prime}=S_{j}$, so $i \neq j$. There is no edge in $\vec{\Delta}$ between $S_{i}$ and $S_{j}$ if and only if $\operatorname{Ext}_{\Lambda}^{1}\left(S_{i} \oplus S_{j}, S_{i} \oplus S_{j}\right)=0$, or equivalently that the module $T={ }_{\Lambda} \Lambda[i, j] \oplus \tau_{\Lambda}^{-} S_{i} \oplus \tau^{-} S_{j}$ is a tilting module. Thus if there is no edge in $\vec{\Delta}$ between $S_{i}$ and $S_{j}$, we obtain that $T_{S_{i}}$ and $T_{S_{j}}$ are contained in a diamond. Conversely, if $T_{S_{i}}$ and $T_{S_{j}}$ are contained in a diamond, then trivially $T={ }_{\Lambda} \Lambda[i, j] \oplus \tau_{\Lambda}^{-} S_{i} \oplus \tau^{-} S_{j}$ by using Corollary [1.2, thus $T$ is a tilting module, and hence there is no edge in $\vec{\Delta}$ between $S_{i}$ and $S_{j}$.

Note that we have the dual statement for different simple $\Lambda$-modules $S, S^{\prime}$, which are not projective, involving the tilting modules $T^{S}$ and $T^{S^{\prime}}$. Also note that in this way we can determine for an arbitrary $\vec{\Delta}$ whether or not there are edges in $\vec{\Delta}$ between non-injective simples and also between non-projective simples.

Given a tilting module $T=\bigoplus_{t=1}^{n} T_{t}$ and $i \neq j$ with $1 \leq i, j \leq n$ we denote it by $T[i, j]=\bigoplus_{t \neq i, j} T_{t}$ 
Lemma 5.3. For $1 \leq i, j \leq n$ let $M={ }_{\Lambda} \Lambda[i, j]$ and assume that $M$ is faithful. Let $T=M \oplus X \oplus Y$ be the unique sink in $l k(M)$. Then:

(1) $X$ and $Y$ are not $\Lambda$-projective.

(2) $\tau_{\Lambda}(X \oplus Y)$ is a tilting module in $M^{\text {perp }}$ which is injective in $M^{\text {perp }}$.

(3) $T^{\prime} \in l k(M)$ if and only if $T^{\prime} \geq T$ in $\mathcal{T}_{\Lambda}$.

Proof. By Theorem 1.4 we have that there is a path of length two from ${ }_{\Lambda} \Lambda$ to $T$ in $\overrightarrow{\mathcal{K}}_{\Lambda}$. By Lemma 1.1 we then have that $X$ and $Y$ are both not projective, thus showing (1). Since $M$ is projective and $M \oplus X \oplus Y$ is a tilting module, we have that $\tau_{\Lambda}(X \oplus Y) \in M^{\text {perp }}$. If $\tau_{\Lambda}(X \oplus Y)$ is not injective in $M^{\text {perp }}$, then there is a connecting sequence in $M^{\text {perp }}$ of the form $0 \rightarrow \tau_{\Lambda} X \rightarrow \widetilde{\tau_{\Lambda} Y} \rightarrow Z \rightarrow 0$ with $\widetilde{\tau_{\Lambda} Y} \in$ add $\tau_{\Lambda} Y$. Since $M$ is faithful $Z$ is not $\Lambda$-injective. But then $M \oplus Y \oplus \tau_{\Lambda}^{-} Z \in \operatorname{lk}(M)$ is a successor of $T$, in contrast to $T$ being the sink in $\operatorname{lk}(M)$, thus showing (2). Finally let $T^{\prime}=M \oplus X_{1} \oplus Y_{1} \in \operatorname{lk}(M)$; then as above $\tau_{\Lambda}\left(X_{1} \oplus Y_{1}\right) \in M^{\text {perp }}$. So by (2) we infer that $\operatorname{Ext}_{\Lambda}^{1}\left(\tau_{\Lambda}\left(X_{1} \oplus Y_{2}\right), \tau_{\Lambda}(X \oplus Y)\right)=0$. But this clearly implies that $T^{\prime} \geq T$. Conversely let $T^{\prime} \in \mathcal{T}_{\Lambda}$ with $T^{\prime} \geq T$. Then $\operatorname{Ext}_{\Lambda}^{1}\left(T^{\prime}, T\right)=0$ shows that $\operatorname{Ext}_{\Lambda}^{1}\left(T^{\prime} \oplus M, T^{\prime} \oplus M\right)=0$; thus $M$ is a direct summand of $T^{\prime}$. So $T^{\prime} \in \operatorname{lk} M$.

We point out that in the situations where we apply Lemma 5.3 the unique sink is combinatorially given. Then Lemma $5.3(3)$ describes the link inside $\mathcal{T}_{\Lambda}$ combinatorially.

Next we will determine which of the neighbors $T_{s}$ of ${ }_{\Lambda} \Lambda$ in $\overrightarrow{\mathcal{K}}_{\Lambda}$ correspond to simple $S$ which are not projective.

Lemma 5.4. Let $\Lambda$ be a hereditary algebra and let $S$ be a simple $\Lambda$-module which is not injective. Then $S$ is not projective if and only if $T_{S}$ occurs in a subquiver of $\overrightarrow{\mathcal{K}}_{\Lambda}$ of the form
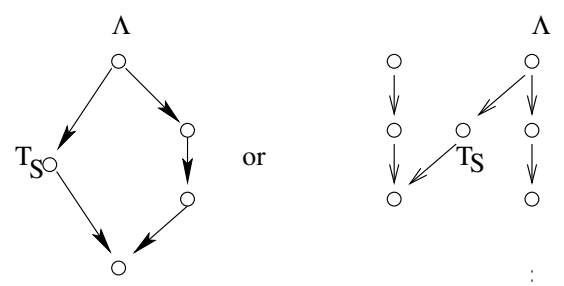

Proof. If $S_{i}=S$ is not projective there is a simple $\Lambda$-module $S_{j}$ with $S_{i} \rightarrow S_{j}$ in $\vec{\Delta}$. Let $M={ }_{\Lambda} \Lambda[i, j]$. Since $S_{i}, S_{j}$ are not injective we have that $M$ is faithful. By Theorem 1.4 we know the shape of $\overrightarrow{\mathcal{K}}_{\Lambda}(M)$. By Lemma 5.2 we know that the diamond cannot occur. Let $T=M \oplus X \oplus Y$ be the unique sink in $\overrightarrow{\mathcal{K}}_{\Lambda}(M)$. We have by Lemma 5.3 that neither $X$ nor $Y$ is a projective $\Lambda$-module and that $\tau_{\Lambda}(X \oplus Y)$ is a tilting module in $M^{\text {perp }}$ which is injective in $M^{\text {perp }}$. Since $S_{i} \in M^{\text {perp }}$ is injective we may assume that $\tau_{\Lambda} X=S_{i}$, or $X=\tau_{\Lambda}^{-} S_{i}$. Let $T^{\prime}$ be the tilting module which lies on the path of length two in $\overrightarrow{\mathcal{K}}_{\Lambda}(M)$ from ${ }_{\Lambda} \Lambda$ to $T$. Then $T^{\prime}$ is of the form $T_{S_{m}}$ 
for some $m$. By Corollary 1.2 we infer that $\tau^{-} S_{m}$ is a direct summand of $T$. Since $\operatorname{Ext}_{\Lambda}^{1}\left(\tau^{-} S_{m}, M\right)=0$ we see that $m=i$ or $m=j$. But $m=j$ is impossible since $\tau^{-} S_{i}$ is a direct summand of $T$ and there is an arrow $S_{i} \rightarrow S_{j}$ in $\vec{\Delta}$. So $m=i$, and thus $T_{S}=T_{S_{i}}$ is in the position as indicated.

Conversely, assume that $T_{S}=T_{S_{i}}$ is contained in a subquiver of $\overrightarrow{\mathcal{K}}_{\Lambda}$ as indicated. The path of length two from ${ }_{\Lambda} \Lambda$ to the unique sink $T$ in the subquiver shows that $T$ is of the form $T={ }_{\Lambda} \Lambda[i, j] \oplus \tau_{\Lambda}^{-} S_{i} \oplus X$ for some $j \neq i$ and a non-projective indecomposable $\Lambda$-module $X$. The position of $T$ in the subquiver shows that $T$ has two predecessors in $\operatorname{lk}\left({ }_{\Lambda} \Lambda[i, j]\right)$. By Theorem 1.4 we see that $T$ is the unique $\operatorname{sink}$ in $\operatorname{lk}\left({ }_{\Lambda} \Lambda[i, j]\right)$ and that ${ }_{\Lambda} \Lambda[i, j]$ is faithful. So we have by Lemma 5.3 that $S_{i} \oplus \tau_{\Lambda} X$ is injective in ${ }_{\Lambda} \Lambda[i, j]^{\text {perp }}$. The subquiver shows that there is at least one more tilting module $T^{\prime}={ }_{\Lambda} \Lambda[i, j] \oplus Z$ such that $\tau_{\Lambda} Z \in M^{\text {perp }}$. Indeed let $T^{\prime}$ be the immediate predecessor of $T$ different from $T_{S}$. The arrow $T^{\prime} \rightarrow T$ in $\overrightarrow{\mathcal{K}}_{\Lambda}$ shows that ${ }_{\Lambda} \Lambda[i, j]$ is a direct summand of $T$, so $T^{\prime}={ }_{\Lambda} \Lambda[i, j] \oplus Z$. If $Z$ has an indecomposable projective direct summand, then $T^{\prime}$ is of the form $T_{S_{m}}$ for some $m$, but $T^{\prime}$ is not an immediate successor of ${ }_{\Lambda} \Lambda$. Thus $\tau_{\Lambda} Z$ is a tilting module in ${ }_{\Lambda} \Lambda[i, j]^{\text {perp }}$ not isomorphic to $S \oplus \tau_{\Lambda} X$. This shows that $M^{\text {perp }}$ is connected, and hence there is an arrow from $S_{i} \rightarrow S_{j}$ in ${ }_{\Lambda} \Lambda[i, j]^{\text {perp }}$, since $S_{i}$ is injective in ${ }_{\Lambda} \Lambda[i, j]^{\text {perp }}$. But then there is an arrow from $S_{i} \rightarrow S_{j}$ in $\vec{\Delta}$, hence $S=S_{i}$ is not projective.

Note that in a dual way we may decide which neighbors of $D \Lambda_{\Lambda}$ in $\overrightarrow{\mathcal{K}}_{\Lambda}$ correspond to simple injective modules.

If $\vec{\Delta}$ is a Dynkin quiver there is a different way to detect in $\overrightarrow{\mathcal{K}}_{\Lambda}$ which neighbors of ${ }_{\Lambda} \Lambda$ correspond to simple projective $\Lambda$-modules.

Proposition 5.5. Let $\Lambda=k \vec{\Delta}$ for a Dynkin quiver $\vec{\Delta}$. Let $S$ be a simple $\Lambda-$ module which is not injective. Then $S$ is projective if and only if $T_{S}$ lies on a path of maximal length in $\overrightarrow{\mathcal{K}}_{\Lambda}$.

Proof. If $S$ is simple projective, then the indecomposable direct summands of $T_{S}$ form a complete slice, so $T_{S}$ lies on a path of maximal length in $\overrightarrow{\mathcal{K}}_{\Lambda}$ by Corollary 4.3. Conversely, assume that $T_{S}$ lies on a path of maximal length in $\overrightarrow{\mathcal{K}}_{\Lambda}$ for a simple $\Lambda$-module $S_{i}=S$. Then the sequence connecting the two complements $P(i), \tau^{-} S_{i}$ of ${ }_{\Lambda} \Lambda[i]$ is the Auslander-Reiten sequence starting at $P(i)$; hence $P(i)=\tau\left(\tau^{-} S_{i}\right)=$ $S_{i}$, so $S_{i}$ is simple projective.

Clearly there is a dual statement for the neighbors of $D \Lambda_{\Lambda}$ in $\overrightarrow{\mathcal{K}}_{\Lambda}$, in case $\overrightarrow{\mathcal{K}}_{\Lambda}$ is finite.

Let $\Lambda$ be a finite dimensional $k$-algebra and $\Lambda^{\prime}=\Lambda \times k$. Let $S$ be the simple $\Lambda^{\prime}$-module corresponding to the factor $k$ in $\Lambda^{\prime}$. Then $\varphi: \mathcal{T}_{\Lambda} \rightarrow \mathcal{T}_{\Lambda^{\prime}}$ defined by $\varphi(T)=T \times S$ is an isomorphism of posets. Thus it is impossible to detect the number of simples unless we assume that $\Lambda$ has no simple projective which is injective. In our situation we equivalently assume that $\vec{\Delta}$ does not have isolated vertices. In Corollary 3.6 we have shown how to determine the number of simple modules in case $\vec{\Delta}$ is connected. We now present a more general result.

Theorem 5.6. Let $\vec{\Delta}$ be a quiver without isolated vertices and $\Lambda=k \vec{\Delta}$. Then the number of simple $\Lambda$-modules is uniquely determined by the neighbors of ${ }_{\Lambda} \Lambda$ and by the neighbors of $D \Lambda_{\Lambda}$ in $\overrightarrow{\mathcal{K}}_{\Lambda}$. 
Proof. By Corollary 3.3 we have that $s\left({ }_{\Lambda} \Lambda\right)=n-\mid$ sources in $\vec{\Delta} \mid$. Let $i$ be a source of $\vec{\Delta}$. By assumption $i$ is not a sink of $\vec{\Delta}$, so by Lemma 5.1 the tilting module $T^{S_{i}}$ is defined and a neighbor of $D \Lambda_{\Lambda}$ is in $\overrightarrow{\mathcal{K}}_{\Lambda}$. By the dual of Lemma 5.4 we can determine the number of neighbors $T^{S}$ of $D \Lambda_{\Lambda}$ corresponding to simple injectives $S$, so we can determine the number of sources and hence we can determine $n$.

Let $S$ be a simple $\Lambda$-module which is not injective. We will consider the subposet defined by $\mathcal{T}_{S}=\left\{T \in \mathcal{T}_{\Lambda} \mid T \leq T_{S}\right\}$. If $S$ is simple injective we set $\mathcal{T}_{S}=\emptyset$. Let $S$ be a simple $\Lambda$-module which is not projective. We define dually a subposet $\mathcal{T}^{S}=\{T \in$ $\left.\mathcal{T}_{\Lambda} \mid T \geq T^{S}\right\}$. If $S$ is projective we set $\mathcal{T}^{S}=\emptyset$. If $S$ is a simple $\Lambda$-module we denote the complement of $\mathcal{T}_{S}$ inside $\mathcal{T}_{\Lambda}$ by $\mathcal{C}\left(\mathcal{T}_{S}\right)=\left\{T \in \mathcal{T}_{\Lambda} \mid T \notin \mathcal{T}_{S}\right\}$. If $S^{\prime}$ is a simple $\Lambda$-module we denote the complement of $\mathcal{T}^{S^{\prime}}$ by $\mathcal{C}\left(\mathcal{T}^{S^{\prime}}\right)=\left\{T \in \mathcal{T}_{\Lambda} \mid T \notin \mathcal{T}^{S^{\prime}}\right\}$. Recall from Lemma 2.3 that for an indecomposable $\Lambda$-module $X$ which is not projective we have defined $T^{\alpha}(X) \in \mathcal{T}_{\Lambda}$. Using left orthogonal categories we can dually define $T_{\omega}(X)$ in case $X$ is not injective.

Proposition 5.7. Let $S, S^{\prime}$ be simple $\Lambda$-modules. Then we have the following for $T \in \mathcal{T}_{\Lambda}$ :

(1) $T \in \mathcal{C}\left(\mathcal{T}_{S}\right)$ if and only if $\operatorname{Hom}_{\Lambda}(T, S) \neq 0$.

(2) $T \in \mathcal{C}\left(\mathcal{T}^{S}\right)$ if and only if $\operatorname{Hom}_{\Lambda}(S, T) \neq 0$.

(3) $\mathcal{C}\left(\mathcal{T}_{S}\right)$ has a unique minimal element. If $S$ is not injective it is of the form $T_{\omega}(S)$

(4) $\mathcal{C}\left(\mathcal{T}^{S}\right)$ has a unique maximal element. If $S$ is not projective it is of the form $T^{\alpha}(S)$.

(5) $\mathcal{C}\left(\mathcal{T}_{S}\right) \cap \mathcal{C}\left(\mathcal{T}^{S}\right)=\left\{T \in \mathcal{T}_{\Lambda} \mid S\right.$ is a direct summand of $\left.T\right\}$.

(6) $\mathcal{C}\left(\mathcal{T}_{S}\right) \cap \mathcal{C}\left(\mathcal{T}^{S^{\prime}}\right) \neq \emptyset$ if and only if $\operatorname{Ext}_{\Lambda}^{1}\left(S^{\prime}, S\right)=0$.

Proof. We first deal with the case that $S$ is injective. Then by definition $\mathcal{C}\left(\mathcal{T}_{S}\right)=\mathcal{T}_{\Lambda}$. So $\operatorname{Hom}_{\Lambda}(T, S) \neq 0$ for $T \in \mathcal{T}_{\Lambda}$ implies $T \in \mathcal{C}\left(\mathcal{T}_{S}\right)$. Conversely let $T \in \mathcal{T}_{\Lambda}$. Since $T$ is sincere we have that $\operatorname{Hom}_{\Lambda}(T, S) \neq 0$. So we may assume that $S$ is not injective. Let $T \in \mathcal{C}\left(\mathcal{T}_{S}\right)$. Then we have that $T_{S} \nsucceq T$, thus $\operatorname{Ext}_{\Lambda}^{1}\left(T_{S}, T\right) \neq 0$; hence by the Auslander-Reiten formula also $\operatorname{Hom}_{\Lambda}(T, S) \neq 0$. Conversely, $\operatorname{Hom}_{\Lambda}(T, S) \neq 0$ implies that $\operatorname{Ext}_{\Lambda}^{1}\left(T_{S}, T\right) \neq 0$, thus $T_{S} \nsupseteq T$, and so $T \in \mathcal{C}\left(\mathcal{T}_{S}\right)$. This shows (1). Clearly (2) is dual to (1).

We will show (4). Clearly (3) is dual to (4). If $S$ is projective, then $\mathcal{C}\left(\mathcal{T}^{S}\right)=\mathcal{T}_{\Lambda}$, so ${ }_{\Lambda} \Lambda \in \mathcal{C}\left(\mathcal{T}^{S}\right)$; hence $\mathcal{C}\left(\mathcal{T}^{S}\right)$ has a unique maximal element. If $S$ is not projective, then it follows from $(2)$ that $T^{\alpha}(S) \in \mathcal{C}\left(\mathcal{T}^{S}\right)$, since $S$ is a direct summand of $T^{\alpha}(S)$. Let $T \in \mathcal{C}\left(\mathcal{T}^{S}\right)$; then by $(2)$ we have that $\operatorname{Hom}_{\Lambda}(S, T) \neq 0$. Since $S$ is simple we have that $S$ is cogenerated by $T$. But then $\operatorname{Ext}_{\Lambda}^{1}(S, T)=0$. The assertion now follows from Lemma 2.3 .

Let $T \in \mathcal{C}\left(\mathcal{T}_{S}\right) \cap \mathcal{C}\left(\mathcal{T}^{S}\right)$ be a tilting module. Thus by (1) and (2) we have that $\operatorname{Hom}_{\Lambda}(T, S) \neq 0$ and that $\operatorname{Hom}_{\Lambda}(S, T) \neq 0$. So $S$ is both generated and cogenerated by $T$. But then $\operatorname{Ext}_{\Lambda}^{1}(T, S)=0$ and $\operatorname{Ext}_{\Lambda}^{1}(S, T)=0$ show that $S$ is a direct summand of $T$. Conversely let $T \in \mathcal{T}_{\Lambda}$ with $S$ a direct summand of $T$. Then $\operatorname{Hom}_{\Lambda}(T, S) \neq 0$ and $\operatorname{Hom}_{\Lambda}(S, T) \neq 0$. So $T \in \mathcal{C}\left(\mathcal{T}_{S}\right) \cap \mathcal{C}\left(\mathcal{T}^{S}\right)$ by (1) and (2). This shows (5).

Let $T \in \mathcal{C}\left(\mathcal{T}_{S}\right) \cap \mathcal{C}\left(\mathcal{T}^{S^{\prime}}\right)$. By $(1)$ and $(2)$ we have that $\operatorname{Hom}_{\Lambda}(T, S) \neq 0$ and that $\operatorname{Hom}_{\Lambda}\left(S^{\prime}, T\right) \neq 0$. So $S$ is generated and $S^{\prime}$ is cogenerated by $T$. So we obtain a surjection $T^{\prime} \rightarrow S$ and an injection $S^{\prime} \rightarrow T^{\prime \prime}$ with $T^{\prime}, T^{\prime \prime} \in$ add $T$. In particular we see that $\operatorname{Ext}_{\Lambda}^{1}(T, S)=0$. This implies that $\operatorname{Ext}_{\Lambda}^{1}\left(S^{\prime}, S\right)=0$. Conversely assume that 
$\operatorname{Ext}_{\Lambda}^{1}\left(S^{\prime}, S\right)=0$. If $S=S^{\prime}$, then trivially $\mathcal{C}\left(\mathcal{T}_{S}\right) \cap \mathcal{C}\left(\mathcal{T}^{S}\right) \neq \emptyset$ by (4) and the fact that $S$ is a partial tilting module. If $S \neq S^{\prime}$, then $S \in S^{\prime \text { perp }}$. If $S^{\prime}$ is projective, then ${ }_{\Lambda} \Lambda \in \mathcal{C}\left(\mathcal{T}_{S}\right) \cap \mathcal{C}\left(\mathcal{T}^{S^{\prime}}\right)$. So we may assume that $S^{\prime}$ is not projective. We consider $T^{\alpha}\left(S^{\prime}\right)=S^{\prime} \oplus P$, where $P$ is a minimal projective generator in $S^{\prime \text { perp }}$. Then $T^{\alpha}\left(S^{\prime}\right) \in \mathcal{C}\left(\mathcal{T}^{S^{\prime}}\right)$ by (4). Since $S \in S^{\prime \text { perp }}$, we clearly have that $\operatorname{Hom}_{\Lambda}(P, S) \neq 0$; hence $T^{\alpha}\left(S^{\prime}\right) \in \mathcal{C}\left(\mathcal{T}_{S}\right)$, thus showing (6).

We point out that Proposition 5.7 also allows us to compute for a given $T \in \mathcal{T}_{\Lambda}$ the number of simple direct summands of $T$.

Corollary 5.8. Let $T \in \mathcal{T}_{\Lambda}$ and $S_{1}, \ldots, S_{n}$ be the simple $\Lambda$-modules. Then the following are equivalent for $I \subset\{1, \ldots, n\}$ :

(1) $T \in\left(\bigcap_{i \in I} \mathcal{C}\left(\mathcal{T}_{S_{i}}\right)\right) \cap\left(\bigcap_{i \in I} \mathcal{C}\left(\mathcal{T}^{S_{i}}\right)\right)$.

(2) $S_{i}$ is a direct summand of $T$ for all $i \in I$.

If we choose $I(T) \subset\{1, \ldots, r\}$ maximal with respect to (1) the cardinality of $I(T)$ gives the number of simple direct summands of $T$.

In connection with the results in section 2 we obtain the promised combinatorial description of the orthogonal categories with respect to simple modules.

Corollary 5.9. Let $\Lambda$ be a hereditary algebra and let $S$ be a simple $\Lambda$-module and $\bmod \Gamma=S^{\text {perp }}$. Then the partially ordered $\operatorname{sets} \mathcal{C}\left(\mathcal{T}_{S}\right) \cap \mathcal{C}\left(\mathcal{T}^{S}\right)$ and $\mathcal{T}_{\Gamma}$ are isomorphic.

If $S$ is a simple $\Lambda$-module which is neither projective nor injective, we have the two vertices $T_{S}$ and $T^{S}$ in $\overrightarrow{\mathcal{K}}_{\Lambda}$. By Lemma 5.4 and its dual we have identified as a set those neighbors of ${ }_{\Lambda} \Lambda$ in $\overrightarrow{\mathcal{K}}_{\Lambda}$ of the form $T_{S}$ and those neighbors of $D \Lambda_{\Lambda}$ of $\overrightarrow{\mathcal{K}}_{\Lambda}$ of the form $T^{S}$. We denote by $S_{r+1}, \ldots, S_{t}$ the simple $\Lambda$-modules which are neither projective nor injective. Next we determine a combinatorial criterion which provides us with the natural bijection between $\left\{T_{S_{i}} \mid r+1 \leq i \leq t\right\}$ and $\left\{T^{S_{i}} \mid r+1 \leq i \leq t\right\}$. Let $S=S_{r+1}$ and $S_{j}$ for some $r+1 \leq j \leq t$. We consider $\mathcal{U}_{j}=\mathcal{C}\left(\mathcal{T}_{S}\right) \cap \mathcal{C}\left(\mathcal{T}^{S_{j}}\right)$ for all $r+1 \leq j \leq t$. If $\mathcal{U}_{j}=\emptyset$, then by Proposition [5.7 we clearly have that $j \neq r+1$. So we may assume that $\mathcal{U}_{j} \neq \emptyset$. Thus $\operatorname{Ext}_{\Lambda}^{1}\left(S_{j}, S\right)=0$. As in Lemma 2.3 we consider the tilting module $T^{\alpha}\left(S_{j}\right)=S_{j} \oplus P_{j}$, where $P_{j}$ is a minimal projective generator of $S_{j}$ perp. By the proof of Proposition [5.7 (6) we have that $T^{\alpha}\left(S_{j}\right) \in \mathcal{U}_{j}$. The following theorem provides us with a combinatorial criterion to determine the natural bijection aimed at the above.

Theorem 5.10. Using the notation above we have that:

(1) $T^{\alpha}\left(S_{j}\right)$ is the unique maximal element in $\mathcal{U}_{j}$.

(2) $j=r+1$ if and only if every immediate successor $T$ of $T^{\alpha}\left(S_{j}\right)$ in $\overrightarrow{\mathcal{K}}_{\Lambda}$ is contained in $\mathcal{C}\left(\mathcal{T}_{S}\right)$.

Proof. (1) follows from Proposition 5.7(4).

Assume that $j=r+1$. So $S_{j}=S$. Let $T$ be an immediate successor of $T^{\alpha}(S)$ in $\overrightarrow{\mathcal{K}}_{\Lambda}$; then $S$ is a direct summand of $T$ by Lemma 2.3. So $\operatorname{Hom}_{\Lambda}(T, S) \neq 0$, thus $T \in \mathcal{C}\left(\mathcal{T}_{S}\right)$ by Proposition 5.7 .

Conversely assume that $j \neq r+1$. Since $\mathcal{U}_{j} \neq \emptyset$ we have by Proposition 5.7 that $\operatorname{Ext}_{\Lambda}^{1}\left(S_{j}, S\right)=0$, hence $S \in S_{j}{ }^{\text {perp }}$. We first show that $S$ is not injective in $S_{j}$ perp. Since $S$ is not injective in $\bmod \Lambda$ there is a simple $S^{\prime}$ with $\operatorname{Ext}_{\Lambda}^{1}\left(S^{\prime}, S\right) \neq 0$. Clearly $S^{\prime} \neq S_{j}$. If $S^{\prime} \in S_{j}{ }^{\text {perp }}$, then this trivially implies that $S$ is not injective in $S_{j}{ }^{\text {perp }}$. Otherwise $\operatorname{Ext}_{\Lambda}^{1}\left(S_{j}, S^{\prime}\right) \neq 0$. Let $0 \rightarrow S^{\prime} \rightarrow Z \rightarrow S_{j}^{\ell} \rightarrow 0$ be the universal 
extension with $\ell=\operatorname{dim} \operatorname{Ext}_{\Lambda}^{1}\left(S_{j}, S^{\prime}\right)$. Applying $\operatorname{Hom}_{\Lambda}\left(S_{j},-\right)$ to this exact sequence shows that $Z \in S_{j}$ perp. Applying $\operatorname{Hom}_{\Lambda}(-, S)$ to this exact sequence shows that $\operatorname{Ext}_{\Lambda}^{1}(Z, S) \simeq \operatorname{Ext}_{\Lambda}^{1}\left(S^{\prime}, S\right)$. Thus $\operatorname{Ext}_{\Lambda}^{1}(Z, S) \neq 0$, and hence $S$ is not injective in $S_{j}^{\text {perp }}$.

As in the proof of Lemma 2.3 we consider the universal extension

$$
0 \rightarrow{ }_{\Lambda} \Lambda \rightarrow Q \rightarrow S_{j}^{\ell} \rightarrow 0
$$

Applying $\operatorname{Hom}_{\Lambda}(-, S)$ to the exact sequence $(*)$ shows that $\operatorname{Hom}_{\Lambda}\left({ }_{\Lambda} \Lambda, S\right) \simeq$ $\operatorname{Hom}_{\Lambda}(Q, S)$. Clearly $\operatorname{Hom}_{\Lambda}\left({ }_{\Lambda} \Lambda, S\right) \simeq k$. Recall that $T^{\alpha}\left(S_{j}\right)=S_{j} \oplus P_{j}$, where $P_{j}$ was multiplicity free with add $P_{j}=$ add $Q$. Now $\operatorname{dim}_{\operatorname{Hom}_{\Lambda}}(Q, S)=1$ implies that there exists a unique indecomposable direct summand $P_{S}$ of $P_{j}$ such that $T^{\alpha}\left(S_{j}\right)=S_{j} \oplus P_{S} \oplus P^{\prime}$ with $\operatorname{Hom}_{\Lambda}\left(P_{S}, S\right) \simeq k$ and $\operatorname{Hom}_{\Lambda}\left(S_{j} \oplus P^{\prime}, S\right)=0$. Note that $P_{S}$ is the projective cover of $S$ in $S_{j}$ perp. Since $S$ is not injective in $S_{j}{ }^{\text {perp }}$, we see that $P^{\prime}$ is faithful in $S_{j}$ perp ; thus by Lemma 1.1 we have that $P_{S}$ is cogenerated by $P^{\prime}$ in $S_{j}^{\text {perp }}$. Then $P_{S}$ is cogenerated by $M=P^{\prime} \oplus S_{j}$ in $\bmod \Lambda$. By construction we have that $\operatorname{Hom}_{\Lambda}(M, S)=0$. Let $0 \rightarrow P_{S} \rightarrow \tilde{M} \rightarrow Y \rightarrow 0$ with $\tilde{M} \in$ add $M$ be the exact sequence connecting the complements $P_{S}$ and $Y$ of $M$. Then $T=M \oplus Y \in \mathcal{T}_{\Lambda}$ is an immediate successor of $T^{\alpha}\left(S_{j}\right)$. Applying $\operatorname{Hom}_{\Lambda}(-, S)$ to this sequence shows that $\operatorname{Hom}_{\Lambda}(Y, S)=0$. Thus $\operatorname{Hom}_{\Lambda}(T, S)=0$, and so $T \notin \mathcal{C}\left(\mathcal{T}_{S}\right)$ by Proposition $5.7(1)$.

\section{THE RECONSTRUCTION}

This section contains the proof the main theorem of this article. The first two lemmas show how to obtain arrows between certain simple $\Lambda$-modules. In the first lemma we will determine the arrows between the simple non-injective $\Lambda$-modules.

Lemma 6.1. Let $\Lambda=k \vec{\Delta}$ be a hereditary algebra. Let $S, S^{\prime}$ be simple $\Lambda$-modules which are not injective. Then there is exactly one arrow $S \rightarrow S^{\prime}$ in $\vec{\Delta}$ if and only if $T_{S}, T_{S^{\prime}}$ occur in a subquiver of $\overrightarrow{\mathcal{K}}_{\Lambda}$ of the form

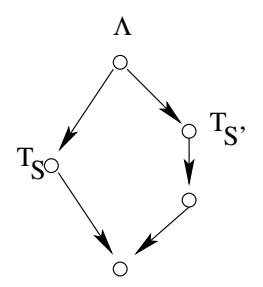

Proof. Let $S_{i}=S$ and $S_{j}=S^{\prime}$. We may assume that $i \neq j$. First assume that $T_{S}$ and $T_{S^{\prime}}$ occur in a subquiver of $\overrightarrow{\mathcal{K}}_{\Lambda}$ as indicated. The unique sink $T$ of the subquiver has to be of the form $T={ }_{\Lambda} \Lambda[i, t] \oplus \tau_{\Lambda}^{-} S_{i} \oplus Y$ for some $t \neq i$ and for some non-projective indecomposable $\Lambda$-module $Y$. By Corollary 1.2 we see that the immediate successor $T^{\prime}$ of $T_{S^{\prime}}$ in this subquiver has to be of the form $T^{\prime}={ }_{\Lambda} \Lambda\left[t^{\prime}, j\right] \oplus \tau_{\Lambda}^{-} S_{j} \oplus Z$ for some $t^{\prime} \neq j$ and for some non-projective indecomposable $\Lambda$-module $Z$. Since there is an arrow $T^{\prime} \rightarrow T$ in $\overrightarrow{\mathcal{K}}_{\Lambda}$ the projective direct summand, ${ }_{\Lambda} \Lambda[i, t]$ of $T$ must be a direct summand of $T^{\prime}$, hence $i=t^{\prime}$ and $t=j$. Moreover the arrow $T^{\prime} \rightarrow T$ in $\overrightarrow{\mathcal{K}}_{\Lambda}$ yields that the tilting modules $T, T^{\prime}$ must have a common direct summand which is an almost complete partial tilting module. Since $S_{i} \not S_{j}$ 
we infer that $Y \simeq Z$. Let $X=Y \simeq Z$. Then it follows that $\tau_{\Lambda}^{-} S_{j}$ and $\tau_{\Lambda}^{-} S_{i}$ are the complements to ${ }_{\Lambda} \Lambda[i, j] \oplus X$ and $\operatorname{dim}_{\operatorname{Ext}_{\Lambda}}^{1}\left(\tau_{\Lambda}^{-} S_{i}, \tau_{\Lambda}^{-} S_{j}\right)=1$ by Lemma 1.1 hence there is exactly one arrow from $S$ to $S^{\prime}$ in $\vec{\Delta}$.

Conversely, assume that there is exactly one arrow $S_{i}=S \rightarrow S_{j}=S^{\prime}$ in $\vec{\Delta}$. Let $M={ }_{\Lambda} \Lambda[i, j]$. Since $S_{i}, S_{j}$ are not injective we have that $M$ is faithful. By Theorem 1.4 we know the shape of $\overrightarrow{\mathcal{K}}_{\Lambda}(M)$. By Lemma 5.2 we know that the diamond cannot occur. Since there is exactly one arrow we see that $\overrightarrow{\mathcal{K}}_{\Lambda}(M)$ is finite. Finally we determine the position of $T_{S_{i}}$ and $T_{S_{j}}$ in $\overrightarrow{\mathcal{K}}_{\Lambda}(M)$. Assume to the contrary that $T_{S_{j}}$ lies on the path of length two from the source ${ }_{\Lambda} \Lambda$ to the sink $T$ in $\overrightarrow{\mathcal{K}}_{\Lambda}(M)$. By Corollary 1.2 we have that $\tau^{-} S_{j}$ is an indecomposable direct summand of $T$. Now $T \leq T_{S_{i}}$ in $\mathcal{T}_{\Lambda}$, thus $\operatorname{Ext}_{\Lambda}^{1}\left(T_{S_{i}}, T\right)=0$. In particular we have that $0=\operatorname{Ext}_{\Lambda}^{1}\left(\tau^{-} S_{i}, \tau^{-} S_{j}\right) \simeq \operatorname{Ext}_{\Lambda}^{1}\left(S_{i}, S_{j}\right)$. But $\operatorname{Ext}_{\Lambda}^{1}\left(S_{i}, S_{j}\right) \neq 0$, since there is an arrow $S_{i} \rightarrow S_{j}$, a contradiction. So $T_{S_{i}}$ and $T_{S_{j}}$ are in the position as indicated.

Dually we obtain the arrows between the simple $\Lambda$-modules which are not projective.

Next we determine whether or not there is an arrow between a simple injective $S$ and a simple projective $S^{\prime}$. Note that the result shown above only used the information given by $\overrightarrow{\mathcal{K}}_{\Lambda}$. If $\Lambda$ is of infinite representation type or equivalently $\mathcal{T}_{\Lambda}$ is infinite, we will use for the next result the full structure given by $\mathcal{T}_{\Lambda}$. The following trivial lemma shows that the existence of arrows from $S^{\prime}$ to $S$ in $\vec{\Delta}$ is deducible from $\mathcal{T}_{\Lambda}$, hence is an invariant of the isomorphism class of $\mathcal{T}_{\Lambda}$.

Lemma 6.2. Let $\Lambda=k \vec{\Delta}$ be a hereditary algebra. Let $S$ be a simple projective $\Lambda$-module and let $S^{\prime}$ be a simple injective $\Lambda$-module. Then there is an arrow $S^{\prime} \rightarrow S$ in $\vec{\Delta}$ if and only if $\mathcal{C}\left(\mathcal{T}_{S}\right) \cap \mathcal{C}\left(\mathcal{T}^{S^{\prime}}\right)=\emptyset$.

Proof. This is a reformulation of Proposition 5.7.

In the proof of the main theorem we have to show whether or not there are multiple arrows between a pair of simple $\Lambda$-modules $S^{\prime}, S$. While Proposition 5.7 . Lemma 6.1 and its dual give a criterion in case $S$ is not projective and $S^{\prime}$ is not injective, the situation seems to be more complicated if $S^{\prime}$ is injective and $S$ is projective. The following lemma will be quite helpful.

Lemma 6.3. Let $\Lambda=k \vec{\Delta}$ be a hereditary algebra. Let $S$ be a simple projective $\Lambda$-module and let $S^{\prime}$ be a simple injective $\Lambda$-module with $\mathcal{C}\left(\mathcal{T}_{S}\right) \cap \mathcal{C}\left(\mathcal{T}^{S^{\prime}}\right)=\emptyset$. Then the following hold:

(1) $\delta\left(T_{S}\right) \geq \delta\left({ }_{\Lambda} \Lambda\right)=t$.

(2) If $\delta\left(T_{S}\right)=\delta\left({ }_{\Lambda} \Lambda\right)$, then there is a unique arrow from $S^{\prime}$ to $S$ in $\vec{\Delta}$.

(3) If $\delta\left(T_{S}\right)=t+s$ for some $s>0$, then there exists a set of neighbors $\left\{M_{1}, \ldots, M_{s}\right\}$ of $T_{S}$ such that $M_{i} \in l k_{\Lambda} \Lambda\left[1, j_{i}\right]$, where $S=S_{1}$ and $S_{j_{i}}$ is simple injective with $\operatorname{Ext}_{\Lambda}^{1}\left(S_{j_{i}}, S\right) \neq 0$. We denote by $I_{S}$ the set of $j_{i}$ occurring in $M_{i}$ for $1 \leq i \leq s$.

(4) $I_{S}=\left\{1 \leq j \leq n \mid S_{j}\right.$ is injective and $\left.\mathcal{C}\left(\mathcal{T}_{S}\right) \cap \mathcal{C}\left(\mathcal{T}^{S_{j}}\right)=\emptyset\right\}$, if $\delta\left(T_{S}\right)>t$.

(5) The set $\left\{M_{1}, \ldots, M_{s}\right\}$ can be combinatorially identified in $\mathcal{T}_{\Lambda}$.

Proof. Since $e\left(T_{S}\right)=1$ it is enough to show that $s\left(T_{S}\right) \geq t-1$. Let $S=S_{1}$ and let $T_{S}=T_{S_{1}}, T_{S_{2}}, \ldots T_{S_{t}}$ be the neighbors of ${ }_{\Lambda} \Lambda$ in $\overrightarrow{\mathcal{K}}_{\Lambda}$. By Lemma $5.1 S_{i}$ is not injective for $1 \leq i \leq t$ and all non-injective simple injective $\Lambda$-modules occur. So 
${ }_{\Lambda} \Lambda[1, i]$ is sincere for $2 \leq i \leq t$, since the projective covers of the simple injective $\Lambda$-modules are direct summands of ${ }_{\Lambda} \Lambda[1, i]$; hence ${ }_{\Lambda} \Lambda[1, i] \oplus \tau_{\Lambda}^{-} S$ is sincere. Thus ${ }_{\Lambda} \Lambda[1, i] \oplus \tau_{\Lambda}^{-} S$ has two complements, yielding neighbors $T_{2}, \ldots, T_{t}$ of $T_{S}$ in $\overrightarrow{\mathcal{K}}_{\Lambda}$. This shows (1).

Assume that $\delta\left(T_{S}\right)=\delta\left({ }_{\Lambda} \Lambda\right)=t$. Let $S_{t+1}=S^{\prime}$. We consider the almost complete partial tilting module ${ }_{\Lambda} \Lambda[1, t+1] \oplus \tau_{\Lambda}^{-} S$. Then $\delta\left(T_{S}\right)=t$ and (1) imply that ${ }_{\Lambda} \Lambda[1, t+1] \oplus \tau_{\Lambda}^{-} S$ has a unique complement $P\left(S^{\prime}\right)$, and so is not sincere by Lemma 1.1. Let $0 \rightarrow S \rightarrow P \rightarrow \tau_{\Lambda}^{-} S \rightarrow 0$ be the Auslander-Reiten sequence starting at $S$. Then $P$ is projective. By assumption $\mathcal{C}\left(\mathcal{T}_{S}\right) \cap \mathcal{C}\left(\mathcal{T}^{S^{\prime}}\right)=\emptyset$, so $\operatorname{Ext}_{\Lambda}^{1}\left(S^{\prime}, S\right) \neq 0$ by Proposition 5.7 But then $\operatorname{Hom}_{\Lambda}\left(\tau_{\Lambda}^{-} S, S^{\prime}\right) \neq 0$. So $\operatorname{Hom}_{\Lambda}\left(P, S^{\prime}\right) \neq 0$, and therefore $P\left(S^{\prime}\right)$ is a direct summand of $P$; hence $\operatorname{Hom}_{\Lambda}\left(P\left(S^{\prime}\right), \tau_{\Lambda}^{-} S\right) \neq 0$. If $S^{\prime \prime}$ is a simple $\Lambda$-module with $S^{\prime \prime} \neq S$, then we also have that $\operatorname{Hom}_{\Lambda}\left(P\left(S^{\prime \prime}\right),{ }_{\Lambda} \Lambda[1, t+1] \oplus \tau_{\Lambda}^{-} S\right) \neq$ 0 . Thus if ${ }_{\Lambda} \Lambda[1, t+1] \oplus \tau_{\Lambda}^{-} S$ is not sincere, then $\operatorname{Hom}_{\Lambda}\left(S,{ }_{\Lambda} \Lambda[1, t+1] \oplus \tau_{\Lambda}^{-} S\right)=0$. But this implies that $P$ is indecomposable. So $P\left(S^{\prime}\right)=P$. So there is exactly one arrow from $S^{\prime}$ to $S$ in $\vec{\Delta}$. This shows (2).

If $\delta\left(T_{S}\right)=t+s$ for some $s>0$ we have the neighbors $T_{2}, \ldots, T_{t}$ constructed in (1). Let $M_{1}, \ldots, M_{s}$ be the different neighbors. Since there is a path of length two from ${ }_{\Lambda} \Lambda$ to $M_{i}$, there is a simple $S_{j_{i}}$ such that $M_{i}={ }_{\Lambda} \Lambda\left[1, j_{i}\right] \oplus \tau_{\Lambda}^{-} S \oplus X_{i}$ for some $X_{i}$ non-projective. Since $M_{i} \notin\left\{T_{2}, \ldots, T_{t}\right\}$ we clearly have that $S_{j_{i}}$ is injective. Since ${ }_{\Lambda} \Lambda\left[1, j_{i}\right] \oplus \tau_{\Lambda}^{-} S$ has the two complements $P\left(j_{i}\right)$ and $X_{i}$, we see that ${ }_{\Lambda} \Lambda\left[1, j_{i}\right] \oplus$ $\tau_{\Lambda}^{-} S$ is sincere. In particular we have that $\operatorname{Hom}_{\Lambda}\left(P\left(S_{j_{i}}\right){ }_{\Lambda} \Lambda\left[1, j_{i}\right] \oplus \tau_{\Lambda}^{-} S\right) \neq 0$. Since $\operatorname{Hom}_{\Lambda}\left(P\left(S_{j_{i}}\right),{ }_{\Lambda} \Lambda\left[1, j_{i}\right]\right)=0$, we have that $\operatorname{Hom}_{\Lambda}\left(P\left(S_{j_{i}}\right), \tau_{\Lambda}^{-} S\right) \neq 0$. But then $\operatorname{Hom}_{\Lambda}\left(P\left(S_{j_{i}}\right), P\right) \neq 0$, where $P$ is the middle term of the Auslander-Reiten sequence starting in $S$. Since $S_{j_{i}}$ is injective this implies that $P\left(S_{j_{i}}\right)$ is a direct summand of $P$, thus $\operatorname{Hom}_{\Lambda}\left(\tau_{\Lambda}^{-} S, S_{j_{i}}\right) \neq 0$; hence $\operatorname{Ext}_{\Lambda}^{1}\left(S_{j_{i}}, S\right) \neq 0$. This shows (3).

It follows from (3) in combination with Proposition 5.7(6) that we have the inclusion $I_{S} \subset\left\{1 \leq j \leq n \mid S_{j}\right.$ is injective and $\left.\mathcal{C}\left(\mathcal{T}_{S}\right) \cap \mathcal{C}\left(\mathcal{T}^{S_{j}}\right)=\emptyset\right\}$.

Conversely, let $j$ be such that $\operatorname{Ext}_{\Lambda}^{1}\left(S_{j}, S\right) \neq 0$ and that $S_{j}$ is injective. Assume that $j \notin I_{S}$. We consider the almost complete partial tilting module $N={ }_{\Lambda} \Lambda[1, j] \oplus$ $\tau_{\Lambda}^{-} S$. Since $j \notin I_{S}$ we have that $N$ is not sincere. Clearly $\operatorname{Hom}_{\Lambda}\left(P\left(S^{\prime}\right), N\right) \neq 0$ for $S^{\prime} \neq S$ and $S^{\prime} \neq S_{j}$. Since $\operatorname{Ext}_{\Lambda}^{1}\left(S_{j}, S\right) \neq 0$, we have that $P\left(S_{j}\right)$ is a direct summand of the middle term $P$ of the Auslander-Reiten sequence starting at $S$; hence $\operatorname{Hom}_{\Lambda}\left(P\left(S_{j}\right), \tau_{\Lambda}^{-} S\right) \neq 0$. So $\operatorname{Hom}_{\Lambda}(S, N)=0$. But then $P=P\left(S_{j}\right)$ is indecomposable. By (3) and the assumption $\delta\left(T_{S}\right)>t$ we have that $I_{S} \neq \emptyset$, and the proof of (3) shows that each element $I_{S}$ gives rise to a direct summand of $P$, a contradiction. So $j \in I_{S}$. This shows (4).

Finally we show assertion (5). Clearly we may assume that $\delta\left(T_{S}\right)=t+s$ for some $s>0$. In (1) and (3) we have constructed two sets of neighbors, namely $\left\{T_{2}, \ldots, T_{t}\right\}$ and $\left\{M_{1}, \ldots, M_{s}\right\}$. The tilting modules $T_{i}$ for $2 \leq i \leq t$ correspond to the neighbors $T_{S_{i}}$ of ${ }_{\Lambda} \Lambda$ different from $T_{S}$. Now ${ }_{\Lambda} \Lambda[1, i]$ is sincere for $2 \leq i \leq t$. Let $K_{i}$ be the sink in $\operatorname{lk}_{\Lambda} \Lambda[1, i]$. We claim that $K_{i}$ is the unique neighbor of $T_{S_{i}}$ such that $T_{S} \geq K_{i}$. Indeed, let $K$ be a neighbor of $T_{S_{i}}$; then $K={ }_{\Lambda} \Lambda[i, j] \oplus \tau_{\Lambda}^{-} S_{i} \oplus X$ for some $j$ and some $X$. Then $T_{S} \geq K$ if and only if $\operatorname{Ext}_{\Lambda}^{1}\left(T_{S}, K\right)=0$, which is equivalent to $\operatorname{Hom}_{\Lambda}(K, S)=0$. But $\operatorname{Hom}_{\Lambda}(K, S)=0$ is equivalent to $j=1$. In this way we can identify the tilting modules $K_{i}$ as distinguished neighbors of $T_{S_{i}}$. We have shown in Lemma 5.3 that $T^{\prime} \geq K_{i}$ for each $T^{\prime} \in \mathrm{lk}_{\Lambda} \Lambda[1, i]$. Since $T_{i} \in \operatorname{lk}_{\Lambda} \Lambda[1, i]$ we see that $T_{i} \geq K_{i}$. Let $1 \leq j \leq s$ and $2 \leq i \leq t$. We claim that 
$M_{j} \geq K_{i}$. By (3) we have that $M_{j}={ }_{\Lambda} \Lambda\left[1, i_{j}\right] \oplus \tau_{\Lambda}^{-} S \oplus X_{j}$ for some simple injective $S_{i_{j}}$. Thus $\operatorname{Ext}_{\Lambda}^{1}\left(M_{j}, P\left(S_{i_{j}}\right)\right) \neq 0$. But $P\left(S_{i_{j}}\right)$ is a direct summand of $K_{i}$, thus $M_{j} \geq K_{i}$. By the previous considerations we have described the set $\left\{K_{2}, \ldots, K_{t}\right\}$ combinatorially. So we obtain from the last two assertions that $\left\{M_{1}, \ldots, M_{s}\right\}=$ $\left\{T \mid T_{S} \rightarrow T\right.$ in $\overrightarrow{\mathcal{K}}_{\Lambda}$ and $T \nsupseteq K_{i}$ for $\left.2 \leq i \leq t\right\}$.

We now present the reconstruction from $\mathcal{T}_{\Lambda}$. To simplify the terminology we define for a quiver $\vec{\Delta}$ the decorated underlying simply-laced quiver $\vec{\Delta}_{\text {dec }}$ as follows. The vertices of both quivers are the same. If for a pair of vertices $(i, j)$ of $\vec{\Delta}$ there is exactly one arrow from $i$ to $j$ in $\vec{\Delta}$, then there is also exactly one arrow from $i$ to $j$ in $\vec{\Delta}_{\text {dec }}$. If for a pair of vertices $(i, j)$ of $\vec{\Delta}$ there are $r \geq 2$ arrows from $i$ to $j$ in $\vec{\Delta}$, then there is exactly one decorated arrow $i \stackrel{*}{\rightarrow} j$ from $i$ to $j$ in $\vec{\Delta}_{\text {dec }}$. So $\vec{\Delta}_{\text {dec }}$ does only carry the information as to whether there are multiple arrows in $\vec{\Delta}$ between two vertices. Trivially, $\vec{\Delta}=\vec{\Delta}_{\text {dec }}$ in the case where there are no multiple arrows in $\vec{\Delta}$.

Theorem 6.4. Let $\vec{\Delta}, \vec{\Delta}^{\prime}$ be two finite quivers without oriented cycles and isolated vertices. Let $\Lambda=k \vec{\Delta}$ and $\Lambda^{\prime}=k \vec{\Delta}^{\prime}$. If $\mathcal{T}_{\Lambda} \simeq \mathcal{T}_{\Lambda^{\prime}}$, then $\vec{\Delta}_{\text {dec }} \simeq \vec{\Delta}_{\text {dec }}^{\prime}$.

Proof. We will show that we can reconstruct $\vec{\Delta}_{\text {dec }}$ from $\mathcal{T}_{\Lambda}$. We will assume that the number of vertices of $\vec{\Delta}$ is $n \geq 2$. By Theorem 5.6 we can determine this number $n$ from $\overrightarrow{\mathcal{K}}_{\Lambda}$. So we know from Corollary 3.3 the number of sources and the number of sinks in $\vec{\Delta}$. We label the simple $\Lambda$-modules $S_{1}, \ldots, S_{r}, S_{r+1}, \ldots, S_{t}, S_{t+1}, \ldots, S_{n}$ such that $S_{i}$ is projective for $1 \leq i \leq r, S_{i}$ is injective for $t+1 \leq i \leq n$ and $S_{i}$ is neither projective nor injective for $r+1 \leq i \leq t$. By Lemma 5.4 we know which of the neighbors of ${ }_{\Lambda} \Lambda$ in $\overrightarrow{\mathcal{K}}_{\Lambda}$ correspond to the simple projective $\Lambda$-modules. Dually we can determine the neighbors of $D \Lambda_{\Lambda}$ which correspond to the simple injective $\Lambda$-modules. By Theorem 5.10 we can combinatorially obtain the natural bijection between the neighbors $T_{S_{i}}$ of $\Lambda \Lambda$ and $T^{S_{i}}$ of $D \Lambda_{\Lambda}$ for $r+1 \leq i \leq t$.

To reconstruct $\vec{\Delta}_{\text {dec }}$ from $\mathcal{T}_{\Lambda}$ we proceed in several steps.

(0) The vertices $\{1, \ldots, n\}$ of $\vec{\Delta}_{\text {dec }}$ correspond bijectively to all neighbors of ${ }_{\Lambda} \Lambda$ in $\overrightarrow{\mathcal{K}}_{\Lambda}$ and certain neighbors of $D \Lambda_{\Lambda}$ in $\overrightarrow{\mathcal{K}}_{\Lambda}$, namely to the set $\left\{T_{S_{i}} \mid 1 \leq i \leq t\right\} \cup$ $\left\{T^{S_{j}} \mid t+1 \leq j \leq n\right\}$, or equivalently by Theorem 5.10 they correspond bijectively to all neighbors of $D \Lambda_{\Lambda}$ in $\overrightarrow{\mathcal{K}}_{\Lambda}$ and certain neighbors of ${ }_{\Lambda} \Lambda$ in $\overrightarrow{\mathcal{K}}_{\Lambda}$, namely to the set $\left\{T^{S_{i}} \mid r+1 \leq i \leq n\right\} \cup\left\{T_{S_{j}} \mid 1 \leq j \leq r\right\}$.

For the determination of the arrows in $\vec{\Delta}_{\text {dec }}$ we distinguish the following three cases.

(1) Let $S_{i}, S_{j}$ be simple $\Lambda$-modules with $1 \leq i, j \leq t$. We are interested in arrows from $j$ to $i$. Thus we can assume that $r+1 \leq j \leq t$. We apply Proposition 5.7. If $\mathcal{C}\left(\mathcal{T}_{S_{i}}\right) \cap \mathcal{C}\left(\mathcal{T}^{S_{j}}\right) \neq \emptyset$, then there is no arrow from $S_{j}$ to $S_{i}$. If $\mathcal{C}\left(\mathcal{T}_{S_{i}}\right) \cap \mathcal{C}\left(\mathcal{T}^{S_{j}}\right)=\emptyset$, then there are arrows from $j$ to $i$. There is exactly one arrow in $\vec{\Delta}_{\text {dec }}$ from $j$ to $i$ if the situation in Lemma 6.1 applies; otherwise we obtain $j \stackrel{*}{\rightarrow} i$ in $\vec{\Delta}_{\text {dec }}$.

(2) Let $S_{i}, S_{j}$ be simple $\Lambda$-modules with $r+1 \leq i, j \leq n$. We are interested in arrows from $j$ to $i$. Thus we can assume that $r+1 \leq i \leq t$. This is clearly dual to (1).

(3) Let $S_{i}, S_{j}$ be simple $\Lambda$-modules with $1 \leq i \leq r$ and $t+1 \leq j \leq n$. We are interested in arrows from $j$ to $i$. Trivially there are no arrows from $i$ to $j$. We 
apply Lemma 6.2 If $\mathcal{C}\left(\mathcal{T}_{S_{i}}\right) \cap \mathcal{C}\left(\mathcal{T}^{S_{j}}\right) \neq \emptyset$, then there is no arrow from $S_{j}$ to $S_{i}$. If $\mathcal{C}\left(\mathcal{T}_{S_{i}}\right) \cap \mathcal{C}\left(\mathcal{T}^{S_{j}}\right)=\emptyset$, then there are arrows from $j$ to $i$. To determine multiple arrows we will use Lemma 6.3

To simplify the notation we set $S=S_{i}$ and $S^{\prime}=S_{j}$. By (0) both $T_{S}$ and $T^{S^{\prime}}$ are identified in $\mathcal{T}_{\Lambda}$. We denote it by $m=\operatorname{dim}_{\operatorname{Ext}_{\Lambda}}^{1}\left(S^{\prime}, S\right)$ and assume that $m>0$.

If $\delta\left(T_{S}\right)=\delta\left({ }_{\Lambda} \Lambda\right)=t$, then by Lemma 6.3 (2) there is exactly one arrow from $S^{\prime}$ to $S$ in $\vec{\Delta}_{\mathrm{dec}}$.

If $\delta\left(T_{S}\right)=t+s$ for some $s>0$, then we obtain from Lemmas 6.3 (3) and 6.3(5) a set of neighbors $\left\{M_{1}, \ldots, M_{s}\right\}$ of $T_{S}$ with $M_{q}={ }_{\Lambda} \Lambda\left[i, j_{q}\right] \oplus \tau_{\Lambda}^{-} S \oplus X_{q}$ for some $t+1 \leq j_{q} \leq n$ with $\operatorname{Ext}_{\Lambda}^{1}\left(S_{j_{q}}, S\right) \neq 0$ and $1 \leq q \leq s$. Since there exists a path of length two from ${ }_{\Lambda} \Lambda$ to $M_{q}$ in $\overrightarrow{\mathcal{K}}_{\Lambda}$, we see that $X_{q}$ is not projective. Let $I_{S}$ be the set of vertices defined and characterized in Lemma 6.3 By Lemma 6.2 we can then determine the set $I_{S}=\left\{j_{q} \mid 1 \leq q \leq s\right\}$. Note that $j \in I_{S}$. We also note that $S \oplus \tau_{\Lambda} X_{q} \in{ }_{\Lambda} \Lambda\left[i, j_{q}\right]^{\text {perp }}$ is a tilting module. Now ${ }_{\Lambda} \Lambda\left[i, j_{q}\right]^{\text {perp }}$ is equivalent to the subcategory of $\bmod \Lambda$ containing those modules which have only composition factors $S$ and $S_{j_{q}}$. In particular ${ }_{\Lambda} \Lambda\left[i, j_{q}\right]^{\text {perp }}$ is closed under submodules. Since $S$ is projective we infer that also $\tau_{\Lambda} X_{q}$ is projective in ${ }_{\Lambda} \Lambda\left[i, j_{q}\right]^{\text {perp }}$, since $S \oplus \tau_{\Lambda} X_{q}$ is a tilting module in ${ }_{\Lambda} \Lambda\left[i, j_{q}\right]^{\text {perp }}$. Since $\operatorname{Ext}_{\Lambda}^{1}\left(S_{j_{q}}, S\right) \neq 0$ we have that ${ }_{\Lambda} \Lambda\left[i, j_{q}\right]^{\text {perp }}$

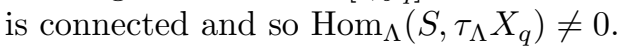

For each $1 \leq q \leq s$ we now consider the subposet $\mathcal{T}_{q}$ of $\mathcal{T}_{\Lambda}$ defined by $\mathcal{T}_{q}=$ $\left\{T \in \mathcal{T}_{\Lambda} \mid T \leq M_{q}\right\}$. Since $\mathcal{T}_{q} \subset \mathcal{T}_{S}$ we see that $\mathcal{C}\left(\mathcal{T}_{S}\right) \subset \mathcal{C}\left(\mathcal{T}_{q}\right)$. We denote by $\mathcal{C}_{q}$ the subposet of $\mathcal{T}_{\Lambda}$ defined by $\mathcal{C}_{q}=\mathcal{C}\left(\mathcal{T}_{q}\right) \backslash \mathcal{C}\left(\mathcal{T}_{S}\right)$. Clearly $\mathcal{C}_{q}=\left\{T \in \mathcal{T}_{\Lambda} \mid \operatorname{Ext}_{\Lambda}^{1}\left(M_{q}, T\right) \neq\right.$ 0 and $S$ is not a direct summand of $T\}$ by Proposition 5.7. Thus $T \in \mathcal{C}_{q}$ if and only if $\operatorname{Hom}_{\Lambda}\left(T, \tau_{\Lambda} M_{q}\right) \neq 0$ and $S$ is not direct summand of $T$. Since $S$ is projective, we have that $\operatorname{Hom}_{\Lambda}(T, S)=0$ if and only if $S$ is not a direct summand of $T$. Thus $T \in \mathcal{C}_{q}$ is equivalent to $\operatorname{Hom}_{\Lambda}\left(T, \tau_{\Lambda} X_{q}\right) \neq 0$ and $S$ is not a direct summand of $T$. We claim that any non-zero map $\phi: T \rightarrow \tau_{\Lambda} X_{q}$ is surjective for $T \in \mathcal{C}_{q}$. Indeed, let $0 \neq \phi: T \rightarrow \tau_{\Lambda} X_{q}$. If $\phi$ is not surjective, we have that $\operatorname{im} \phi$ is a proper submodule of $\tau_{\Lambda} X_{q}$, and so is contained in ${ }_{\Lambda} \Lambda\left[i, j_{q}\right]^{\text {perp }}$. Since $\tau_{\Lambda} X_{q}$ is projective in ${ }_{\Lambda} \Lambda\left[i, j_{q}\right]^{\text {perp }}$ we infer that $\operatorname{im} \phi \in$ add $S$. But then $S$ is a direct summand of $T$, in contrast to $T \in \mathcal{C}_{q}$. Thus $\phi$ is surjective. This implies that $\operatorname{Ext}_{\Lambda}^{1}\left(T, \tau_{\Lambda} X_{q}\right)=0$ for each $T \in \mathcal{C}_{q}$.

We now consider for each $1 \leq q \leq s$ the subposet $\mathcal{U}_{q}=\mathcal{C}_{q} \cap \mathcal{C}\left(\mathcal{T}^{S^{\prime}}\right)$. We claim that $\mathcal{U}_{q}=\emptyset$ for all $1 \leq q \leq s$ if and only if $m \geq 2$.

Indeed, assume first that there is a tilting module $T \in \mathcal{U}_{q}$ for some $q$. By the previous considerations we have that $\operatorname{Ext}_{\Lambda}^{1}\left(T, \tau_{\Lambda} X_{q}\right)=0$, since $T \in \mathcal{C}_{q}$, and by Proposition 5.7 that $S^{\prime}$ is a direct summand of $T$, since $T \in \mathcal{C}\left(\mathcal{T}^{S^{\prime}}\right)$. Thus also $\operatorname{Ext}_{\Lambda}^{1}\left(S^{\prime}, \tau_{\Lambda} X_{q}\right)=0$. Consider the projective resolution

$$
0 \rightarrow K^{\prime} \rightarrow P\left(S^{\prime}\right) \rightarrow S^{\prime} \rightarrow 0 \text { of } S^{\prime} .
$$

Since $\operatorname{Ext}_{\Lambda}^{1}\left(S^{\prime}, S\right) \neq 0$ we have that $S$ is a direct summand of $K^{\prime}$. If $S_{j_{q}} \neq S^{\prime}$, then $S^{\prime}$ is not a composition factor of $\tau_{\Lambda} X_{q}$, so $\operatorname{Hom}_{\Lambda}\left(P\left(S^{\prime}\right), \tau_{\Lambda} X_{q}\right)=0$. This yields by applying $\operatorname{Hom}_{\Lambda}\left(-, \tau_{\Lambda} X_{q}\right)$ to $(*)$ that $\operatorname{Hom}_{\Lambda}\left(K^{\prime}, \tau_{\Lambda} X_{q}\right) \simeq \operatorname{Ext}_{\Lambda}^{1}\left(S^{\prime}, \tau_{\Lambda} X_{q}\right)$. Since $S$ is a direct summand of $K^{\prime}$ and $\operatorname{Hom}_{\Lambda}\left(S, \tau_{\Lambda} X_{q}\right) \neq 0$, we see that $\operatorname{Ext}_{\Lambda}^{1}\left(S^{\prime}, \tau_{\Lambda} X_{q}\right) \neq 0$, a contradiction. Thus $S_{j_{q}}=S^{\prime}$. In particular, $S^{\prime} \in{ }_{\Lambda} \Lambda\left[i, j_{q}\right]^{\text {perp }}$. Since $\operatorname{Ext}_{\Lambda}^{1}\left(S^{\prime}, \tau_{\Lambda} X_{q}\right)$ $=0$ this clearly implies that $m=1$.

Conversely, assume that $m=1$. We have $j \in I_{S}$ for $S^{\prime}=S_{j}$ by Lemma 6.3(4) and our assumption that $\operatorname{Ext}_{\Lambda}^{1}\left(S^{\prime}, S\right) \neq 0$. So $j=j_{q}$ for some $1 \leq q \leq s$. Let 
$M_{q}={ }_{\Lambda} \Lambda[i, j] \oplus \tau_{\Lambda}^{-} S \oplus X_{q}$. Since $m=1$, we have that $S^{\prime}$ and $\tau_{\Lambda} X_{q}$ are both injective in ${ }_{\Lambda} \Lambda[i, j]^{\text {perp }}$, so $S^{\prime} \oplus \tau_{\Lambda} X_{q}$ is a partial tilting module in $\bmod \Lambda$. So there is a tilting module $T=S^{\prime} \oplus \tau_{\Lambda} X_{q} \oplus C$ for some $C$. Trivially $T \in \mathcal{C}\left(\mathcal{T}^{S^{\prime}}\right)$. Since $\operatorname{Ext}_{\Lambda}^{1}\left(S^{\prime}, S\right) \neq 0$ we see that $S$ is not a direct summand of $T$. Since $\tau_{\Lambda} X_{q}$ is a direct summand of $T$, we then see that $T \in \mathcal{C}_{q}$; thus $T \in \mathcal{U}_{q}$. So there is $q$ such that $\mathcal{U}_{q} \neq \emptyset$.

The last assertion now enables us to determine if $m \geq 2$. If for each $1 \leq q \leq s$ the subposet $\mathcal{U}_{q}=\emptyset$, then we obtain in $\vec{\Delta}_{\text {dec }}$ a decorated arrow $S^{\prime} \stackrel{*}{\rightarrow} S$. Otherwise there is exactly one arrow from $S^{\prime}$ to $S$ in $\vec{\Delta}_{\text {dec }}$.

This finishes the proof of the theorem.

Corollary 6.5. Let $\vec{\Delta}$ be a quiver without oriented cycles. If $\vec{\Delta}$ does not contain multiple arrows and isolated vertices, then $\Lambda=k \vec{\Delta}$ is uniquely determined (up to isomorphism) by $\mathcal{T}_{\Lambda}$.

Corollary 6.6. Let $\vec{\Delta}$ be a Dynkin quiver without isolated vertices. Then $\vec{\Delta}$ is uniquely determined (up to isomorphism) by $\overrightarrow{\mathcal{K}}_{\Lambda}$.

Proof. Since $\overrightarrow{\mathcal{K}}_{\Lambda}$ is finite the partially ordered set $\mathcal{T}_{\Lambda}$ is determined by $\overrightarrow{\mathcal{K}}_{\Lambda}$, since it is easily seen that $T \leq T^{\prime}$ in $\mathcal{T}_{\Lambda}$ implies the existence of a path from $T$ to $T^{\prime}$ in $\overrightarrow{\mathcal{K}}_{\Lambda}$ by applying 2.2 of [HU2]. Thus the result follows from Theorem 6.4 .

We also mention the following corollary.

Corollary 6.7. Let $\vec{\Delta}$ be a finite quiver without oriented cycles and isolated vertices. Then $\mathcal{T}_{\Lambda}$ determines whether or not $\vec{\Delta}$ is connected.

Proof. Indeed, we can reconstruct $\vec{\Delta}_{\text {dec }}$ from $\mathcal{T}_{\Lambda}$. But $\vec{\Delta}_{\text {dec }}$ is connected if and only if $\vec{\Delta}$ is connected.

\section{REFERENCES}

[AHT] I. Assem, D. Happel, S. Trepode, Extending tilting modules to one-point extensions by projectives, Comm. in Algebra 35 (2007), no. 10, 2983-3006. MR2356133

[AR] M. Auslander, I. Reiten, Applications of contravariantly finite subcategories, Adv. Math. 86 (1991), 111-152. MR.1097029 (92e:16009)

[ARS] M. Auslander, I. Reiten, S. Smalø, Representation theory of artin algebras, Cambridge University Press, 1995. MR.1314422 (96c:16015)

[GL] Geigle, W., Lenzing, H., Perpendicular categories with applications to representations and sheaves, J. Algebra 144, 273-343 (1991). MR.1140607 (93b:16011)

[H1] D. Happel, Partial tilting modules and recollement, Proceedings of the International Conference of Algebra, Contemporary Mathematics 131, Providence 1992, 345-362. MR.1175843 (93k:16011)

[H2] D. Happel, Selforthogonal modules, Abelian Groups and Modules, Kluwer Academic Publishers, 257-276. MR1378204 (97d:16016)

[HR] D. Happel, C. M. Ringel, Tilted algebras, Trans. Amer. Math. Soc., 274 (1982), 399-443. MR675063 (84d:16027)

[HU1] D. Happel, L. Unger, On a partial order of tilting modules, Algebr. Represent. 8 (2005), no. 2, 147-156. MR2162278(2006e:16021)

[HU2] D. Happel, L. Unger, On the quiver of tilting modules, J. Algebra 284 (2005). no. 2, 857-868. MR2114583 (2005j:16013)

[HU3] D. Happel, L. Unger, Minimal elements in the poset of tilting modules, Contemp. Math. 376, Amer. Math. Soc., Providence, RI, 2005, 281-288. MR 2147028 (2006k:16025)

[R] C. M. Ringel, Tame algebras and integral quadratic forms, Springer Lecture Notes in Mathematics 1099, Heidelberg, 1984. MR774589 (87f:16027) 
[U1] L. Unger, On the simplicial complex of tilting modules over quiver algebras, Proc. London Math. Soc. (3) 73 (1996), 27-46. MR.1387082 (97g:16019)

[U2] L. Unger, On the simplicial complex of exceptional modules, Habilitationsschrift, Universität Paderborn, 1993.

Fakultät für Mathematik, Technische Universität Chemnitz, D-09107 Chemnitz, GerMANY

E-mail address: happel@mathematik.tu-chemnitz.de

Fakultät für Mathematik und Informatik, Fernuniversität Hagen, D-58084 Hagen, Germany

E-mail address: luise.unger@fernuni-hagen.de 